\title{
Commensal Streptococcus mitis produces two different lipoteichoic acids of type I and type IV
}

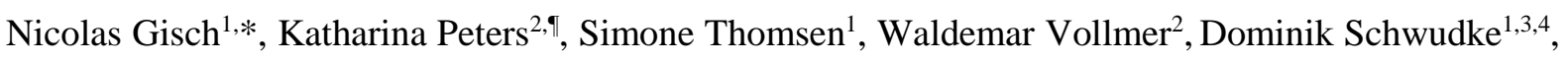
and Dalia Denapaite ${ }^{5,8}$

${ }^{1}$ Division of Bioanalytical Chemistry, Priority Area Infections, Research Center Borstel, Leibniz Lung Center, 23845 Borstel, Germany

${ }^{2}$ Centre for Bacterial Cell Biology, Biosciences Institute, Newcastle University, NE2 4AX Newcastle upon Tyne, UK

${ }^{3}$ German Center for Infection Research (DZIF), Thematic Translational Unit Tuberculosis, Partner Site: Hamburg-Lübeck-Borstel-Riems, 23845 Borstel, Germany

${ }^{4}$ Airway Research Center North, Member of the German Center for Lung Research (DZL), 23845 Borstel, Germany.

${ }^{5}$ Department of Microbiology, University of Kaiserslautern, 67663 Kaiserslautern, Germany

IIPresent address: School of Natural \& Environmental Sciences, Faculty of Science, Agriculture \& Engineering, Newcastle University, Newcastle upon Tyne, NE1 7RU, UK.

${ }^{\S}$ Present address: Department of Cellular, Computational and Integrative Biology, University of Trento, Trento, Italy

\author{
*Corresponding author: Nicolas Gisch \\ E-mail: ngisch@fz-borstel.de
}

Running title: Lipoteichoic acids of S. mitis

Keywords: Glycosylglycerolipids; Lipoteichoic acid; Mass spectrometry (MS); Nuclear magnetic resonance (NMR); Streptococcus mitis. 


\begin{abstract}
The opportunistic pathogen Streptococcus mitis possesses, like other members of the Mitis group of viridans streptococci, phosphorylcholine ( $P$-Cho)-containing teichoic acids (TAs) in its cell wall. Bioinformatic analyses predicted the presence of TAs that are almost identical with those identified in the pathogen S. pneumoniae, but a detailed analysis of $S$. mitis lipoteichoic acid (LTA) was not performed to date. Here we determined the structures of LTA from two S. mitis strains, the highlevel beta-lactam and multiple antibiotic resistant strain B6 and the penicillin-sensitive strain NCTC10712. In agreement with bioinformatic predictions we found that the structure of one LTA (type IV) was like pneumococcal LTA, except the exchange of a glucose moiety with a galactose within the repeating units. Further genome comparisons suggested that the majority of $S$. mitis strains should contain the same type IV LTA as $S$. pneumoniae, providing a more complete understanding of the biosynthesis of these $P$-Cho-containing TAs in members of the Mitis group of streptococci. Remarkably, we observed besides type IV LTA an additional polymer belonging to LTA type I in both investigated S. mitis strains. This LTA consists of $\beta$-galactofuranosyl-(1,3)-diacylglycerol as glycolipid anchor and a poly-glycerol-phosphate chain at the $O-6$ position of the furanosidic galactose. Hence, these bacteria are capable of synthesizing two different LTA polymers, most likely produced by distinct biosynthesis pathways. Our bioinformatics analysis revealed the prevalence of the LTA synthase LtaS, most probably responsible for the second LTA version (type I), amongst $S$. mitis and $S$. pseudopneumoniae strains.
\end{abstract}




\section{INTRODUCTION}

Streptococcus mitis is a member of the Mitis group of viridans streptococci $(1,2)$ and is closely related to S. pneumoniae, S. pseudopneumoniae, S. oralis and S. infantis species (3). The primary habitat of these species is the human upper respiratory tract. $S$. mitis is rarely associated with diseases and presents mostly a non-virulent behavior $(3,4)$. Nevertheless, it can become an opportunistic pathogen via entering the bloodstream and causing bacteremia and/or infective endocarditis (5-7). In contrast, $S$. pneumoniae, which is the closest relative of $S$. mitis, is a well-known respiratory pathogen that is capable of causing a broad spectrum of diseases such as otitis media, bacteremia, pneumonia, and meningitis in humans $(8,9)$. Analyses of the evolution of members of the Mitis group based on genome comparison suggested that $S$. mitis, S. pseudopneumoniae and S. pneumoniae arose from a common ancestor, which was a pneumococcus-like organism (10). Both species evolved in parallel either with adaptation to commensal lifestyle (S. mitis) by losing virulence-associated genes and genome reduction, or by improving the genomic plasticity necessary for a pathogenic lifestyle $(S$. pneumoniae $)(3,11)$. The species S. pseudopneumoniae appears to be genetically intermediary between these two species and was first described in $2004(11,12)$.

Lipoteichoic acids (LTA) are main constituents of the Gram-positive cell wall. Their precise role in bacterial physiology has not been fully elucidated, but some LTA-deficient mutants display attenuated virulence in mouse models, defects in biofilm formation or cell division $(13,14)$. In general, LTA contains a lipophilic anchor formed by diacyl-glycerol (DAG), which anchors these molecules to the cell membrane. At the $O-3$ position, DAG carries a glycosyl moiety with attached complex backbone structures consisting of repetitive units (RUs), which are highly variable between different Grampositive bacteria. Five general LTA types are currently known and are mainly characterized by the architecture of their RUs: polyglycerol-phosphate (type I), complex glycosyl-glycerol-phosphate (type II + III), glycosyl-ribitol-phosphate (type IV), or glycosyl-phosphate (type V) (13). Recently, we showed that the pig pathogen $S$. suis expresses typical type I LTA molecules and complex, mixed-type LTAs, which contain a combination of RUs present in type I and type II/III LTA (15). Type I LTA, the most common and best characterized polymer, has a simple structure consisting of a poly-glycerolphosphate (poly-Gro-P) chain anchored to a glycolipid in the membrane (13). This type of LTA is found 
in a many Firmicutes, such as Bacillus subtilis, Staphylococcus aureus, Listeria monocytogenes and S. agalactiae $(13,16)$. Type I LTA was also identified in a Streptococcus sp. strain DSM 8747 with the rare glycolipid anchor 3-O-( $\beta$-D-galactofuranosyl)-1,2-diacylglycerol. DSM 8747 is described as closely related to S. pneumoniae (17) although its taxonomic characterization is unpublished and thus the genetic distance to $S$. pneumoniae remained unclear. S. pneumoniae produces structurally unique and very complex type IV LTA (18-20). Similar structures are only known for other members of Mitis group of streptococci, such as S. mitis SK137 (structure of WTA has been described (21)) and S. oralis Uo5 (22). Unlike other bacteria, the TAs in these bacteria and potentially additional members of the Mitis group (such as S. pseudopneumoniae and S. infantis) share a common biosynthetic pathway as predicted by bioinformatics (23) showing identical chains of both TA polymers (14,19). Remarkably, S. mitis B6 also has a gene encoding a homolog of the LTA synthase LtaS (23). LtaS mediates the polymerization of GroP chains from the head group of the membrane lipid phosphatidylglycerol onto the glycolipid anchor in LTA type I biosynthesis $(24,25)$. The number of LtaS-type enzymes involved in type I LTA synthesis differs between bacterial species. In S. aureus LtaS initiates and extends the GroP chain, whereas in L. monocytogenes the LTA primase LtaP links the first GroP to the glycolipid anchor, after which LtaS extends the chain (13). This raises the question whether $S$. mitis can produce both type I and type IV LTA. However, up to now the $S$. mitis LTA has not been analyzed.

Here, we report the comprehensive structural analysis of LTA isolated from two S. mitis strains, the high-level beta-lactam and multiple antibiotic resistant strain B6 (26) and the penicillin-sensitive strain NCTC10712 $(27,28)$. We determined the LTA structures by chemical degradation, highresolution mass spectrometry, and one- and two-dimensional, homo- and heteronuclear NMR spectroscopy. We also isolated and analyzed the glycolipids of S. mitis B6, verifying the presence of three different glycosylglycerolipids. Furthermore, we assessed key TA biosynthesis genes in the genomes of selected members of the Mitis group of streptococci. 


\section{RESULTS}

\section{Isolation and structural analysis of LTA from S. mitis B6 and NCTC10712}

LTA from the two S. mitis strains B6 and NCTC10712 were prepared according to our previously published workflow for structural analysis (14). LTA shows a tendency to form large aggregates and micelles in water (29) and we showed for S. pneumoniae LTA that the first sugar moieties bound to the diacyl-glycerol are not detectable in NMR spectra (18). Therefore, we used $O$ deacylated (hydrazine-treated) and non-aggregating LTA molecules for our analysis. Fig. 1 shows the comparison of ${ }^{1} \mathrm{H}$ NMR spectra of $O$-deacylated LTA from the two $S$. mitis strains and $S$. pneumoniae. Spectra have been recorded in deuterated $25 \mathrm{mM}$ sodium phosphate buffer ( $\mathrm{pH} \mathrm{5.5)} \mathrm{at} 300 \mathrm{~K}$. The adjustment of the $\mathrm{pH}$ value of the NMR solvent was necessary for a homogeneous degree of protonation of the amino group at position 4 of the 2-acetamido-4-amino-2,4,6-trideoxygalactose (AATGalp), making the NMR chemical shifts better comparable and reproducible between multiple samples. The spectra of $O$-deacylated LTA from the two $S$. mitis strains were almost identical but slightly different to the spectrum of $O$-deacylated LTA from $S$. pneumoniae strain D39 $\Delta c p s \Delta l g t$ (this strain lacks the capsular polysaccharide and the gene encoding for the lipoprotein diacylglyceryl transferase (Lgt) and is therefore deficient in lipidation of pre-lipoproteins; its LTA has been shown to be identical with that of the D39 wild type $(14,18)$ ), indicating different composition in type IV LTA (see dashed lines in magnification of the anomeric region). Interestingly, the $S$. mitis spectra also showed the presence of an additional polymer that was absent in S. pneumoniae and is represented, e.g., by an additional anomeric proton at $\delta_{\mathrm{H}} 5.02 \mathrm{ppm}$ (black arrows, Fig. 1) and intensive signals between $\delta_{\mathrm{H}} 4.10-3.85 \mathrm{ppm}$ (marked with *, Fig. 1). Further NMR analyses then proved the presence of a second type of LTA. In addition to the ribitol-phosphate containing LTA known from pneumococci, the NMR signals identified a glycerol-phosphate containing polymer. The best approach to compare these classes is by their ${ }^{31} \mathrm{P} \mathrm{NMR}$ spectra (Fig. 2). The broad signal in the ${ }^{31} \mathrm{P}$ NMR spectra from 1.48-1.12 ppm (B6) or 1.39-1.10 ppm (NCTC10712) was only present in $O$-deacylated LTA from $S$. mitis, coming from poly-glycerolphosphate chains. The shift of signals for the phosphates in ribitol-5-P moieties from 1.89/1.80 ppm in pneumococcal $O$-deacylated LTA to $1.64 / 1.52 \mathrm{ppm}(\mathrm{B} 6)$ and $1.61 / 1.48 \mathrm{ppm}$ (NCTC10712) in $S$. mitis 
further points to a deviating carbohydrate content in the type IV LTA. The same chemical shifts for the phosphates of the $P$-Cho moieties reflect the identity of the structure in this part of the molecules. Fig. 3 shows the ${ }^{1} \mathrm{H},{ }^{13} \mathrm{C}$-heteronuclear single quantum correlation (HSQC) NMR of LTA $\mathrm{N} 2 \mathrm{H} 4_{4}$ of $S$. mitis strain B6 and the signal assignment as an example for both strains, since almost identical spectra were obtained. The complete NMR chemical shift data for type I and type IV O-deacylated $S$. mitis LTA are summarized in Tables 1 and 2. The $O$-deacylated lipid anchor of the type IV LTA is $\alpha$-glucopyranosylglycerol. The following RUs all have the pseudo-pentasaccharide composition $(\rightarrow 4)-6-O-P$-Cho- $\alpha$-DGal $p$ NAc- $(1 \rightarrow 3)-6-O-P-C h o-\beta-D-G a l p N A c-(1 \rightarrow 1)-R i b-o l-5-P-(O \rightarrow 6)-\beta-$ D-Gal $p-(1 \rightarrow 3)-$ $\operatorname{AATGal} p(1 \rightarrow)$. The first RU (RU 1) attached to the lipid anchor is $\beta$-linked, whereas all other RUs, representing an elongation of the carbohydrate part in the LTA molecule, are $\alpha$-1-linked to the precedent RU. The $O$-deacylated lipid anchor of the type I LTA is $\beta$-galactofuranosyl-glycerol, which is elongated by the poly-glycerol-phosphate chain at the $O-6$ position of the $\mathrm{Gal}_{f}$.

The two LTA polymers are present in almost equally amounts in both strains. As judged by integration of the ${ }^{1} \mathrm{H}$ NMR signals for $\mathrm{Gal}_{f} \mathrm{H}-1$ (for type I LTA) and C' $\mathrm{H}-1$ (for type IV LTA) the ratio was appr. 55:45 in both preparations of strain NCTC10712. The ratio in strain B6 was in one preparation 60:40, in the other 40:60. To verify the two different LTA types, we analyzed the $O$-deacylated LTA preparations from the $S$. mitis strains using high-resolution mass spectrometry. Due to very different ionization efficiencies of the two LTA types, we adjusted the recorded mass range in a way that each LTA type was monitored separately. Type I LTA molecules ionized as doubly or triply charged species and were recorded in an $m / z$-window of 500 to 1650 . By contrast, type IV LTA molecules ionized as quadruple and quintuple charged species and were recorded in an $\mathrm{m} / z$-window of 1600 to 3000 . Representative charge deconvoluted spectra are depicted in Fig. 4. The identified molecular species are listed in Tables 3 and 4, respectively. The LTA of strain NCTC10712 lacks the molecular species displaying a complete $P$-Cho substitution pattern, the most abundant molecules present lack one $P$-Cho substituent (Fig. 4B). This is due to a more pronounced $P$-Cho hydrolysis of the terminal RU mediated by the phosphorylcholine esterase Pce, which is also indicated by the shift of the ${ }^{1} \mathrm{H}$ NMR signal representing the anomeric proton of the terminal $\alpha$-D-6-O-P-Cho-Gal $p$ NAc $\left(\mathbf{E}^{\text {term }}\right)$ from $\delta_{\mathrm{H}} 5.08 \mathrm{ppm}$ 
to $\delta_{\mathrm{H}} 5.07 \mathrm{ppm}$ (Fig. 1, right panel). We recently reported that Pce is the only enzyme that modifies the $P$-Cho substitution pattern of the TAs in S. pneumoniae (30). This effect was not observed in a second, independent LTA preparation of this strain. In this second preparation less $P$-Cho hydrolysis and a tendency to longer chain length was observed (Fig. S1). However, these differences did not impact the general structural composition of the LTA. Finally, the ${ }^{1} \mathrm{H}$ NMR analysis of the native LTA preparation of S. mitis NCTC10712 (Fig. S2) revealed the presence of alanine residues on the LTA molecules, as is observed in S. pneumoniae. The chemical structures of the two LTA polymers identified in the two analyzed S. mitis strains are shown in Fig. 5.

\section{Investigation of the glycosylglycerolipids from $S$. mitis B6}

As they represent the putative cell wall anchor motifs for LTA molecules, we also isolated the glycosylglycerolipids from $S$. mitis strain B6 by preparative thin-layer chromatography. The ${ }^{1} \mathrm{H}$ NMR spectra of the pool of mono-glycosyl-diacylglycerols and di-glycosyl-diacylglycerols are depicted in Fig. 6, respectively. Our analysis revealed the presence of two different mono-glycosyl-diacylglycerols, $\alpha$-glucopyranosyl-(1,3)-diacylglycerol and $\beta$-galactofuranosyl-(1,3)-diacylglycerol, and a single diglycosyl-diacylglycerol, $\quad \alpha$-galactopyranosyl-(1,2)- $\alpha$-glucopyranosyl-(1,3)-diacylglycerol. $\quad$ The complete NMR chemical shift data for the identified glycosylglycerolipids are summarized in Table 5.

\section{In silico analysis of LTA biosynthesis key enzymes in S. mitis and closely related species}

The TAs in members of the Mitis group like S. mitis, S. pneumoniae and S. oralis share a common biosynthetic pathway (23), which therefore have structurally identical carbohydrate chains in both of their TAs - LTA and WTA - and the analysis of one of the polymers can be considered as representative for both. For S. mitis only the structural analysis of the WTA of strain SK137 has been described. In this strain a $\beta$-D-glucose has been identified within the RU (21), which was identical to that in S. pneumoniae $(14,18)$. Our structural analysis of the LTA of S. mitis strains B6 and NCTC10712 revealed the presence of a $\beta$-D-galactose at this position, which was in line with the expectation based on the gene content of these strains. Hence, we raised the question about the predominant structural 
type in S. mitis strains. BLAST searches in the NCBI microbial genomes database revealed that $68.8 \%$ of the $S$. mitis strains (95 out of 138 strains) possessed the equivalent of the S. mitis B6 smi_1983 gene, which shows 94\% homology to sp70585_0164 of S. pneumoniae 70585 (Table S1.1). The S. mitis type strain NCTC12261 (also named ATCC 49456) is identical to most S. mitis strains in this respect. 36 strains (26.8\%) were identical to Spr0091/SPD_0098 of S. pneumoniae R6/D39 and therefore likely incorporate a glucose into their TA. S. mitis SK137 strain shows homology to Spr0091 (97\% identity), which is consistent with the reported structure (21). Only seven S. mitis strains (5.1\%) did not reveal an identical gene, which is probably due to incompleted genome sequences or a potential mislabeling of these strains. Indeed six out of seven of these genomes do not contain any genes encoding proteins involved in the biosynthesis of type IV LTA (Table S1.1), hence these were not further considered in this study.

In order to ascertain the homogeneity of the $S$. mitis group, we focused for further analysis on four different enzymes, two from S. mitis B6 and two from S. oralis Uo5, which are essential for the biosynthesis of the respective type IV TA and searched for homologs by tBlastn analysis. The selected enzymes were the teichoic acid flippase TacF (Smi_1229; Spr1150 in S. pneumoniae R6 (23)) and the repeating unit (RU) polymerase (Smi_0770; Spr1222 in S. pneumoniae R6 (23)) for S. mitis. As determinants for an S. oralis-like TA structure we chose the S. oralis Uo5 enzymes TacF (Sor_0765) and LicD4 (Sor_0762). The latter has no homolog encoded from the S. mitis/S. pneumoniae genomes. LicD4 is a large membrane protein (717 aa) with two defined regions. The N-terminal region is predicted to have 12 transmembrane helices and might catalyze the polymerization of the TA precursors, the C-terminal LicD-type region adds at least one phosphorylcholine to the precursor chain repeating units $(22,23)$. S. oralis Uo5 TacF shares only $47 \%$ sequence identity with TacF of $S$. mitis/S. pneumoniae (23). Our analysis revealed that four of the 138 strains do not contain the S. mitis but the S. oralis type IV LTA synthesis genes, consequently they are likely $S$. oralis strains (Table S1.1). Interestingly, for 12/132 strains hits with >80\% identity to TacF of both types were observed. 10 of 12 of these strains also contained the S. mitis RU-polymerase Smi_0770, in the two remaining strains neither the gene for Smi_0770 nor for LicD4 of S. oralis (Sor_0762) was present. For 42 of the 132 (31.8\%) strains designated as $S$. mitis only a hit for S. oralis TacF was observed with high sequence 
identity (>80\%). This suggests that S. mitis has more structural diversity in type IV TAs than has been previously anticipated.

The presence of two LTA molecules (types I and IV) in the analyzed S. mitis strains prompted us to investigate three aspects in detail. As the bioinformatics analysis indicated the presence of an LtaS-homolog of S. aureus in the S. mitis B6 (Smi_0753) genome (23), it is most likely that in S. mitis LtaS facilitates the synthesis of type I LTA. Therefore, we first searched for the corresponding ltaS gene in the genome of S. mitis NCTC10712 strain. Both proteins, Smi_0753 (of strain B6) and SMI10712_00646 (of strain NCTC10712), have 97\% residues in common and are predicted to be polytopic membrane proteins with 5-transmembrane segments and a large C-terminal extracellular loop containing the complete sulfatase domain (PF00884), as does the LtaS protein (SAV0719) of S. aureus (24). Second, we used the S. mitis Smi_0753 protein sequence as query in tBlastn searches against the S. mitis genomes available in NCBI microbial genome database (Table S1.1). Most S. mitis strains (100 out of $132,75.8 \%$ ) contain an LtaS homolog with $98 \%$ to $91 \%$ sequence identity. The 32 S. mitis genomes in which ltaS was absent contained the genes encoding proteins involved in the biosynthesis of $S$. oralis-like type IV TA, except three strains (Table S1.2). Third, we searched for LtaS homologs in related species, but we found no LtaS orthologs encoded by the $S$. pneumoniae, $S$. oralis and $S$. infantis genomes. This finding is in agreement with previous data $(23,31)$, however 85 of 111 deposited S. pseudopneumoniae genomes (76.6\%) contained an encoded ltaS gene (Table S2). For the remainder of 26 strains lacking the ltaS gene, 25 were not identified as $S$. pseudopneumoniae strains in the most recent phylogenetic analysis (32) (Table S2). All strains assigned as S. pseudopneumoniae contained the LtaS protein with a sequence identity of $>97 \%$ to LtaS of S. mitis B6. Taken together, our genome analysis shows that LtaS homologs are present only in S. mitis and S. pseudopneumoniae. To further corroborate this, we selected representative strains from $S$. pneumoniae, $S$. mitis and $S$. pseudopneumoniae and analyzed the genomic localization of ltaS. We selected strains with available information about TA structures for four S. pneumoniae and three S. mitis strains (B6, NCTC10712, and SK137) as well as the type strains S. mitis NCTC12261 and S. pseudopneumoniae ATCC BAA960. S. pseudopneumoniae IS7493 was selected due to the presence of a completed genome sequence. The ltaS gene is present as a monocistronic operon in S. mitis and S. pseudopneumoniae genomes (Fig. 
7). All S. pneumoniae strains show a similar structure of this locus but lack the ltaS gene. The upstream and downstream regions are well conserved in all three species showing 90-93\% identity at the nucleotide level. In addition, a BoxC element is located in the intergenic region between spr1236 and spr1237 genes in the S. pneumoniae genomes. Box elements are short repeated DNA sequences that are distributed randomly within the S. pneumoniae genomes (33). The function and origin of Box elements are still unknown, but they were found to enhance the genetic diversity and genome plasticity in $S$. pneumoniae (34).

We also searched for putative promoter regions and putative transcriptional terminators of the ltaS gene in the B6 genome. Surprisingly, we found that the ltaS gene has two start codons, which are well conserved in all selected strains except S. pneumoniae (Fig. 8). It has been shown that in bacteria the predominant initiation codon is ATG $(80.1 \%)$, while the alternative codon GTG is rarely occurring (11.6\%) (35). However, for both start codons we distinctly predicted the promoter regions (Figure 8). The presence of two putative promoter regions and two putative translation start codons suggests a complex regulation of ltaS expression. The first promoter (start codon GTG) contains a -10 region, the putative ribosomal biding site, but the -35 element is not well conserved and is difficult to find. The second promoter (transcriptional start codon ATG) exhibits an extended -10 promoter element, which is four times more common in S. pneumoniae than in E. coli and can function without a -35 element (36). Remarkably, the four S. pneumoniae genomes contain the promoter region, although the ltaS gene is missing. Most likely, S. pneumoniae has lost the ltaS gene during evolution.

\section{DISCUSSION}

The present study provides the first detailed structural analysis of LTA molecules isolated from S. mitis. For our analysis, we have chosen the penicillin-sensitive strain NCTC10712 and the high-level beta-lactam resistant as well as multiple antibiotic resistant strain B6 as representatives. This structural investigation complements our earlier bioinformatics analysis of the TA-biosynthesis genes in members of the Mitis group (23), which suggested that the LTA of S. oralis should have an overall architecture similar to pneumococcal LTA with certain alterations due to a divergent enzymatic repertoire. We previously presented the $S$. oralis LTA structure and finally linked the observed structural variations 
between S. oralis and S. pneumoniae LTA to the distinct enzymes for TA biosynthesis (22). Based on these analyses $(22,23)$ we predicted that $S$. mitis contains a type IV LTA with similar structure to that of $S$. pneumoniae (18), since LTA biosynthesis genes and their organization in the genomes of $S$. pneumoniae (R6) and S. mitis (B6) are almost identical (94-99\% homology) with only one exception. The smi1983 gene located in an operon equivalent to spr0091-spr0092 encodes a glycosyltransferase that is similar to S. pneumoniae SP70585_0164 and S. oralis Sor_1862. The gene sp70585_0164 was identified in the genome of the S. pneumoniae Sp70585 serotype 5 strain, which has been shown to incorporate galactose instead of glucose into its WTA (37) and Sor_1862 incorporates galactose into $S$. oralis LTA (22). The recently discovered lipoteichoic acid ligase TacL, which mediates the last step in LTA biosynthesis in S. pneumoniae (14) is conserved in both S. mitis strains with 97\% homology. In summary, this suggested that $S$. mitis B6 contains a type IV LTA like pneumococci but with a galactose instead of the $\alpha$-glucose. We confirmed this prediction by the structural analysis of the S. mitis LTA. Based on the BLAST search we predict that a subset of $S$. mitis strains with a homolog of Spr0091/SPD_0098 from S. pneumoniae R6/D39 contain exactly the same type IV LTA as $S$. pneumoniae (Table S1). This is consistent with results of a recent study (11), which showed that the majority of $S$. mitis strains possessed the equivalent of the $S$. pneumoniae 70585 gene SP70585_RS00795, as do all S. oralis and S. infantis strains, but it is exceptional amongst the $S$. pneumoniae strains. Moreover, two of the three identified glycosylglycerolipids of the S. mitis cell wall, $\alpha$-glucopyranosyl-(1,3)-diacylglycerol $\quad$ and $\quad \alpha$-galactopyranosyl-(1,2)- $\alpha$-glucopyranosyl-(1,3)diacylglycerol, are identical to those present in S. pneumoniae (38). By contrast, the third identified glycosylglycerolipid, $\beta$-galactofuranosyl-(1,3)-diacylglycerol, is not present in $S$. pneumoniae. Even more remarkable, a second LTA polymer belonging to structure type I containing exactly this molecule as lipid anchor is present in both $S$. mitis strains investigated here. Besides $S$. suis (15), this is the second description of a Gram-positive bacterium being capable of synthezising two different LTA polymers. In the case of $S$. mitis the different LTAs are most likely synthezised via two distinct biosynthesis pathways, whereas in S. suis the synthesis of the two identified LTA types seems to be based on a single biosynthesis route. The type I LTA characterized in this study has been reported earlier in a Streptococcus sp. strain DSM 8747 (17), but the taxonomic characterization has never been published, 
thus the true genetic relatedness to S. pneumoniae remains unclear. Our finding of a type I LTA polymer in S. mitis strains contrasts an observation of Hogg et al (39) who suggested the presence of polyglycerol-phosphate containing LTA in many oral streptococci, but not in S. oralis and S. mitis strains based on the detection of these molecules in phenol extracts of lyophilized cells using a poly-glycerolphosphate-specific monoclonal antibody. A recent study (40) suggested the presence of a type I LTA besides the type IV LTA in S. mitis based on the identification of a potential biosynthetical intermediate, glycerophospho-diglycosyl-diacylglycerol (GPDGDAG). However, the authors of that study also reported the identification of GPDGDAG in S. pneumoniae and S. oralis, which do not contain type I LTA $(18,22,41)$, what is further corroborated by the absence of LtaS in these species as elucidated here. Moreover, the mentioned work of Wei et al (40) showed by using an LtaS-knockout of S. mitis strain ATCC 49456 (SM61; NCTC12261) that GPDGDAG synthesis does not require LtaS. Notably, Wei et al could not identify, as earlier works (39), type I LTA by a poly-glycerol-phosphate-specific monoclonal antibody (40). We now clearly provide the missing evidence for a type I LTA in S. mitis by isolating this LTA and its subsequent structural analysis. Since the detected glycolipid anchor in the observed type I LTA is a mono-galactofuranosyl-DAG its synthesis will be independent from GPDGDAG synthesis, because this molecule contains a diglycosyl moiety and does not contain a galactofuranose. Our bioinformatics analysis aiming to identify the putative 1,2-diacylglycerol-3- $\beta$ galactofuranosyl transferase (or mono-galactofuranosyl-diacylglycerol synthase) involved in LTA glycolipid anchor biosynthesis in the $S$. mitis genomes did not provide a candidate enzyme. To the best of our knowledge such an enzyme has not been described in other bacteria so far.

Our bioinformatics analyses identified ltaS homologs only in S. mitis and S. pseudopneumoniae genomes, which distinguishes these from closely related species like S. pneumoniae, S. oralis and $S$. infantis. This is especially interesting regarding the human pathogen $S$. pneumoniae, which is genetically much closer related to $S$. mitis and $S$. pseudopneumoniae than $S$. oralis and $S$. infantis. The detailed and manual comparison of genomes done here is based on a limited number of genomes and requires as critical prerequisite the correct species assignment, which can be problematic, as seen by the recently reported misidentification of $S$. pseudopneumoniae $(11,32)$. Here we selected specific LTA 
biosynthesis genes for an S. mitis/S. pneumoniae or an S. oralis-like LTA structure. At the current stage, this does not allow an unequivocal assignment of isolates to a specific species, which would require a higher number of curated genome sequences and the investigation of the associated TA structures. Therefore, future studies should focus on potentially new S. mitis subgroups, e.g., those containing homologs of both S. mitis-and S. oralis-type TacF, as well as the recently identified S. oralis subspecies. The higher structural diversity in TA biosynthesis within $S$. mitis has also been addressed recently by Kilian \& Tettelin (11).

In summary, our work sets the stage for further investigations on the biosynthesis pathway of this particular type I LTA in S. mitis, the role of LtaS in its biosynthesis, and functional studies. Our work further suggests that a defined set of LTA biosynthesis genes could become a valuable metric to better differentiate between the closely related species studied here and more distant species such as $S$. infantis and the $S$. oralis subspecies tigurinus and dentisanii.

\section{EXPERIMENTAL PROCEDURES}

\section{Bacterial strains and growth conditions}

S. mitis B6 and NCTC10712 strains were grown at $37{ }^{\circ} \mathrm{C}$ without aeration in tryptone soya broth (TSB; Oxoid) medium and growth was followed by monitoring the absorbance at $600 \mathrm{~nm}$. The strains were grown on D-agar plates supplemented with $3 \%$ defibrinated sheep blood (42). Cell wall preparation of S. mitis was performed as described previously (22). Briefly, bacteria were usually cultured in 5-liter batches of TSB medium harvested at late exponential phase by centrifugation $(7,500 \times \mathrm{g}, 10 \mathrm{~min})$ at room temperature. The cell pellet was washed with citric buffer $(50 \mathrm{mM}, \mathrm{pH} 4.7)$ and resuspended in citric buffer containing $4 \%$ sodium dodecyl sulfate. The cell suspension was incubated for $20 \mathrm{~min}$ at $100{ }^{\circ} \mathrm{C}$, stored at $-80{ }^{\circ} \mathrm{C}$ and lyophilized subsequently.

\section{Extraction and isolation of LTA}

LTA purification was performed as described elsewhere (14) twice independently for each strain. Yields of LTA preparations from 5 liters of bacterial culture were: for strain B6 $4.9 \mathrm{mg}$ and $12.1 \mathrm{mg}$; for strain NCTC10712: $4.9 \mathrm{mg}$ (4 L culture) and $10.2 \mathrm{mg}$. 


\section{Chemical treatments of LTA}

Hydrazin treatment (to yield $O$-deacyl LTA) was performed following our earlier described procedure (15).

\section{Isolation of glycosylglycerolipids}

For glycosylglycerolipids extraction, $126 \mathrm{mg}$ dry bacterial pellet $S$. mitis strain B6 were suspended in 2 mL Millipore-water and $7.5 \mathrm{~mL} \mathrm{CHCl} / 3 / \mathrm{MeOH} 1: 2 \mathrm{v} / \mathrm{v}$ and thoroughly mixed (in a $50 \mathrm{~mL}$ Nalgene Oak Ridge Centrifuge Tube (FEP), Thermo Scientific). After $1 \mathrm{~h}$ shaking at RT $2.5 \mathrm{~mL}$ Millipore-water and $2.5 \mathrm{~mL} \mathrm{CHCl}_{3}$ were added, mixed and shaken for another hour at RT. In parallel, for each of the four isolation batches $2 \mathrm{~mL}$ Millipore-water were treated in the same way to generate "equilibrated water". The isolation mixtures were centrifuged at $4,000 \times g$ for 10 minutes at $4{ }^{\circ} \mathrm{C}$ and the organic phases were transferred in fresh tubes. The remaining aqueous phase/interphase was extracted again with the organic phase of the "equilibrated water"-generation (10 s mixing, centrifugation as above). Resulting organic phases were combined with those of the first extractions. These combined phases were than washed with $4 \mathrm{~mL}$ of the "equilibrated water" (10 s mixing; centrifugation as above). The resulting organic phases were collected and dried under a stream of nitrogen (yielding $4.85 \mathrm{mg}$ crude glycolipid extract). Glycosylglycerolipids were separated in two portions (applied as $5 \mu \mathrm{g} / \mu \mathrm{L}$-solution in $\mathrm{CHCl}_{3} / \mathrm{MeOH}$ 2:1 v/v) by high-performance thin-layer chromatography (HPTLC) in $\mathrm{CHCl}_{3} / \mathrm{MeOH} 85: 15 \mathrm{v} / \mathrm{v}$ on glassbacked $10 \times 10 \mathrm{~cm}$ silica gel $60 \mathrm{~F}_{254}$ plates (Merck) and a small sidebar of the plate was stained with Hanessian's stain (0.5 g of cerium(IV) sulphate tetrahydrate and $25 \mathrm{~g}$ of ammonium molybdate tetrahydrate in $471 \mathrm{ml}$ of water supplemented with $29 \mathrm{ml}$ of sulfuric acid with stirring (43)) at $150{ }^{\circ} \mathrm{C}$. Appropriate bands were scrapped off from the silica $\left(\mathrm{R}_{\mathrm{f}}\right.$ : 0.28-0.38, di-glycosyl-diacylglycerol; 0.560.65, mono-glycosyl-diacylglycerols) and recovered by extraction $\mathrm{CHCl}_{3} / \mathrm{MeOH} 2: 1 \mathrm{v} / \mathrm{v}$ (30 s thorough mixing and subsequent centrifugation $\left(1,080 \times g, 10 \mathrm{~min}, 4^{\circ} \mathrm{C}\right.$; three rounds of collecting and replacing the organic supernatant). After drying under a stream of nitrogen, the residual silica was removed by filtration in $\mathrm{CHCl}_{3} / \mathrm{MeOH} 2: 1 \mathrm{v} / \mathrm{v}$ using a Acrodisc CR $13 \mathrm{~mm}$ Syringe Filter $(0.2 \mu \mathrm{m}$ PTFE membrane, PALL Life Sciences; washed with 2 x $2 \mathrm{~mL} \mathrm{CHCl}_{3} / \mathrm{MeOH}$ 2:1 v/v prior to use). 


\section{NMR spectroscopy}

Deuterated solvents were purchased from Deutero GmbH (Kastellaun, Germany). NMR spectroscopic measurements were performed in $\mathrm{D}_{2} \mathrm{O}$ or deuterated $25 \mathrm{mM}$ sodium phosphate buffer $(\mathrm{pH} 5.5$; to suppress fast de-alanylation) at $300 \mathrm{~K}$ on a Bruker Avance ${ }^{\mathrm{III}} 700 \mathrm{MHz}$ (equipped with an inverse $5 \mathrm{~mm}$ quadruple-resonance Z-grad cryoprobe or with an inverse $1.7 \mathrm{~mm}$ triple-resonance Z-grad micro cryoprobe $)$. Acetone was used as an external standard for calibration of ${ }^{1} \mathrm{H}\left(\delta_{\mathrm{H}}=2.225\right)$ and ${ }^{13} \mathrm{C}\left(\delta_{\mathrm{C}}=\right.$ 30.89) NMR spectra (44) and $85 \%$ of phosphoric acid was used as an external standard for calibration of ${ }^{31} \mathrm{P}$ NMR spectra $\left(\delta_{\mathrm{P}}=0.00\right)$. Analysis of glycosylglycerolipids was performed in $\mathrm{CD}_{3} \mathrm{OD}$ and spectra were calibrated using the residual solvent peak $\left(\delta_{\mathrm{H}}=3.31, \delta_{\mathrm{C}}=49.0\right)(44)$. All data were acquired and processed by using Bruker TOPSPIN V 3.1 or higher. ${ }^{1} \mathrm{H}$ NMR assignments were confirmed by 2D ${ }^{1} \mathrm{H},{ }^{1} \mathrm{H}-\mathrm{COSY}$ and total correlation spectroscopy (TOCSY) experiments. ${ }^{13} \mathrm{C}$ NMR assignments were indicated by $2 \mathrm{D}{ }^{1} \mathrm{H},{ }^{13} \mathrm{C}-\mathrm{HSQC}$, based on the ${ }^{1} \mathrm{H}$ NMR assignments. Interresidue connectivity and further evidence for ${ }^{13} \mathrm{C}$ assignment were obtained from $2 \mathrm{D}{ }^{1} \mathrm{H},{ }^{13} \mathrm{C}$-heteronuclear multiple bond correlation and ${ }^{1} \mathrm{H},{ }^{13} \mathrm{C}-\mathrm{HSQC}-\mathrm{TOCSY}$. Connectivity of phosphate groups were assigned by $2 \mathrm{D}{ }^{1} \mathrm{H},{ }^{31} \mathrm{P}-\mathrm{HMQC}$ and ${ }^{1} \mathrm{H},{ }^{31} \mathrm{P}-\mathrm{HMQC}-\mathrm{TOCSY}$.

\section{Mass spectrometry}

All samples were measured on a Q Exactive Plus mass spectrometer (Thermo Scientific, Bremen, Germany) using a Triversa Nanomate (Advion, Ithaca, NY) as ion source. All measurements were performed in negative-ion mode using a spray voltage of $-1.1 \mathrm{kV}$. Samples were dissolved in a water/propan-2-ol/trimethylamine/acetic acid mixture (50:50:0.06:0.02, v/v/v/v) in a final concentration of appr. $0.13 \mathrm{mg} / \mathrm{mL}$. The mass spectrometer was externally calibrated with glycolipids of known structure. All mass spectra were charge deconvoluted and given mass values refer to the monoisotopic mass of the neutral molecules, if not indicated otherwise. Deconvoluted spectra were computed using Xtract module of Xcalibur 3.1. Software (Thermo, Bremen, Germany). 


\section{Bioinformatic tools and analysis}

The streptococcal genomic sequences used for the identification of genes involved in TA biosynthesis in the study are listed in Table S3 together with NCBI accession numbers and references. The accession numbers of the other S. mitis and S. pseudopneumoniae genomes used in this study are listed in Tables S1 and S2, respectively. In total 140 S. mitis and 112 S. pseudopneumoniae genomes were available in NCBI microbial genome database (as of 3rd January 2021). Two S. mitis strains (SK136 and NCTC12261) and S. pseudopneumoniae type strain ATCC BAA-960 (also known as CCUG 49455) were sequenced and deposited twice. Therefore, 138 different S. mitis and 111 S. pseudopneumoniae strains were included in the tBlastn analysis (45). The putative promoter regions were predicted by two consensus hexamers: -35 (5'-TTGACA-3`) and -10 (5'-TATAAT-3`) located upstream of the translation start codons of ltaS. Rho-independent transcriptional terminator was identified by Mfold program accessed through the mfold Web Server (46). Transmembrane helices and domain structure were predicted by using SMART Tool (http://smart.embl-heidelberg.de/) (47).

\section{Data availability}

All data are contained within the manuscript.

Acknowledgements: We gratefully acknowledge H. Käßner and B. Kunz (both RCB) for excellent technical assistance. We are indebted to N. Frankenberg-Dinkel for providing laboratory facilities to carry out the work of D.D. We would like to thank Reinhold Brückner for supplying the strains and helpful discussions. The work of D.D. was supported by the Deutsche Forschungsgemeinschaft (grant HA1011/11-3).

Conflict of interest: The authors declare that they have no conflicts of interest with the contents of this article. 


\section{REFERENCES}

1. Whatmore, A. M., Efstratiou, A., Pickerill, A. P., Broughton, K., Woodard, G., Sturgeon, D., George, R., and Dowson, C. G. (2000) Genetic relationships between clinical isolates of Streptococcus pneumoniae, Streptococcus oralis, and Streptococcus mitis: characterization of "Atypical" pneumococci and organisms allied to S. mitis harboring S. pneumoniae virulence factor-encoding genes. Infect Immun 68, 1374-1382

2. Bishop, C. J., Aanensen, D. M., Jordan, G. E., Kilian, M., Hanage, W. P., and Spratt, B. G. (2009) Assigning strains to bacterial species via the internet. BMC Biol 7, 3

3. Kilian, M., Riley, D. R., Jensen, A., Brüggemann, H., and Tettelin, H. (2014) Parallel evolution of Streptococcus pneumoniae and Streptococcus mitis to pathogenic and mutualistic lifestyles. MBio 5, e01490-01414

4. Mitchell, J. (2011) Streptococcus mitis: walking the line between commensalism and pathogenesis. Mol Oral Microbiol 26, 89-98

5. Matsui, N., Ito, M., Kuramae, H., Inukai, T., Sakai, A., and Okugawa, M. (2013) Infective endocarditis caused by multidrug-resistant Streptococcus mitis in a combined immunocompromised patient: an autopsy case report. $J$ Infect Chemother 19, 321-325

6. Shelburne, S. A., Sahasrabhojane, P., Saldana, M., Yao, H., Su, X., Horstmann, N., Thompson, E., and Flores, A. R. (2014) Streptococcus mitis strains causing severe clinical disease in cancer patients. Emerg Infect Dis 20, 762-771

7. Rasmussen, L. H., Højholt, K., Dargis, R., Christensen, J. J., Skovgaard, O., Justesen, U. S., Rosenvinge, F. S., Moser, C., Lukjancenko, O., Rasmussen, S., and Nielsen, X. C. (2017) In silico assessment of virulence factors in strains of Streptococcus oralis and Streptococcus mitis isolated from patients with Infective Endocarditis. J Med Microbiol 66, 1316-1323

8. Kadioglu, A., Weiser, J. N., Paton, J. C., and Andrew, P. W. (2008) The role of Streptococcus pneumoniae virulence factors in host respiratory colonization and disease. Nat Rev Microbiol 6, 288-301

9. Henriques-Normark, B., and Tuomanen, E. I. (2013) The pneumococcus: epidemiology, microbiology, and pathogenesis. Cold Spring Harb Perspect Med 3, a010215

10. Kilian, M., Poulsen, K., Blomqvist, T., Håvarstein, L. S., Bek-Thomsen, M., Tettelin, H., and Sørensen, U. B. S. (2008) Evolution of Streptococcus pneumoniae and its close commensal relatives. PLoS One 3, e2683

11. Kilian, M., and Tettelin, H. (2019) Identification of virulence-associated properties by comparative genome analysis of Streptococcus pneumoniae, S. pseudopneumoniae, $S$. mitis, three $S$. oralis subspecies, and S. infantis. MBio 10, e01985-01919

12. Arbique, J. C., Poyart, C., Trieu-Cuot, P., Quesne, G., Carvalho Mda, G., Steigerwalt, A. G., Morey, R. E., Jackson, D., Davidson, R. J., and Facklam, R. R. (2004) Accuracy of phenotypic and genotypic testing for identification of Streptococcus pneumoniae and description of Streptococcus pseudopneumoniae sp. nov. J Clin Microbiol 42, 46864696

13. Percy, M. G., and Gründling, A. (2014) Lipoteichoic acid synthesis and function in Gram-positive bacteria. Annu Rev Microbiol 68, 81-100

14. Heß, N., Waldow, F., Kohler, T. P., Rohde, M., Kreikemeyer, B., Gómez-Mejia, A., Hain, T., Schwudke, D., Vollmer, W., Hammerschmidt, S., and Gisch, N. (2017) Lipoteichoic acid deficiency permits normal growth but impairs virulence of Streptococcus pneumoniae. Nat Commun 8, 2093

15. Gisch, N., Auger, J.-P., Thomsen, S., Roy, D., Xu, J., Schwudke, D., and Gottschalk, M. (2018) Structural analysis and immunostimulatory potency of lipoteichoic acids 
isolated from three Streptococcus suis serotype 2 strains. J Biol Chem 293, 1201112025

16. Rajagopal, M., and Walker, S. (2017) Envelope structures of Gram-positive bacteria. Curr Top Microbiol Immunol 404, 1-44

17. Roethlisberger, P., Iida-Tanaka, N., Hollemeyer, K., Heinzle, E., Ishizuka, I., and Fischer, W. (2000) Unique poly(glycerophosphate) lipoteichoic acid and the glycolipids of a Streptococcus sp. closely related to Streptococcus pneumoniae. Eur J Biochem 267, 5520-5530

18. Gisch, N., Kohler, T., Ulmer, A. J., Müthing, J., Pribyl, T., Fischer, K., Lindner, B., Hammerschmidt, S., and Zähringer, U. (2013) Structural reevaluation of Streptococcus pneumoniae lipoteichoic acid and new insights into its immunostimulatory potency. $J$ Biol Chem 288, 15654-15667

19. Gisch, N., Peters, K., Zähringer, U., and Vollmer, W. (2015) The pneumococcal cell wall. in Streptococcus pneumoniae: Molecular mechanisms of host-pathogen interactions (Brown, J. S., Hammerschmidt, S., and Orihuela, C. J. eds.), Elsevier, UK. pp 145-167

20. Behr, T., Fischer, W., Peter-Katalinić, J., and Egge, H. (1992) The structure of pneumococcal lipoteichoic acid. Improved preparation, chemical and mass spectrometric studies. Eur J Biochem 207, 1063-1075

21. Bergström, N., Jansson, P.-E., Kilian, M., and Skov Sørensen, U. B. (2000) Structures of two cell wall-associated polysaccharides of a Streptococcus mitis biovar 1 strain. A unique teichoic acid-like polysaccharide and the group $\mathrm{O}$ antigen which is a $\mathrm{C}$ polysaccharide in common with pneumococci. Eur J Biochem 267, 7147-7157

22. Gisch, N., Schwudke, D., Thomsen, S., Heß, N., Hakenbeck, R., and Denapaite, D. (2015) Lipoteichoic acid of Streptococcus oralis Uo5: a novel biochemical structure comprising an unusual phosphorylcholine substitution pattern compared to Streptococcus pneumoniae. Sci Rep 5, 16718

23. Denapaite, D., Brückner, R., Hakenbeck, R., and Vollmer, W. (2012) Biosynthesis of teichoic acids in Streptococcus pneumoniae and closely related species: lessons from genomes. Microb Drug Resist 18, 344-358

24. Gründling, A., and Schneewind, O. (2007) Synthesis of glycerol phosphate lipoteichoic acid in Staphylococcus aureus. Proc Natl Acad Sci U S A 104, 8478-8483

25. Reichmann, N. T., and Gründling, A. (2011) Location, synthesis and function of glycolipids and polyglycerolphosphate lipoteichoic acid in Gram-positive bacteria of the phylum Firmicutes. FEMS Microbiol Lett 319, 97-105

26. Denapaite, D., Brückner, R., Nuhn, M., Reichmann, P., Henrich, B., Maurer, P., Schähle, Y., Selbmann, P., Zimmermann, W., Wambutt, R., and Hakenbeck, R. (2010) The genome of Streptococcus mitis B6 - what is a commensal? PLoS One 5, e9426

27. Kilian, M., Mikkelsen, L., and Henrichsen, J. (1989) Taxonomic study of viridans streptococci: description of Streptococcus gordonii sp. nov. and emended descriptions of Streptococcus sanguis (White and Niven 1946), Streptococcus oralis (Bridge and Sneath 1982), and Streptococcus mitis (Andrewes and Horder 1906). Int J Syst Evol Microbiol 39, 471-484

28. Hakenbeck, R., Balmelle, N., Weber, B., Gardès, C., Keck, W., and de Saizieu, A. (2001) Mosaic genes and mosaic chromosomes: intra- and interspecies genomic variation of Streptococcus pneumoniae. Infect Immun 69, 2477-2486

29. Courtney, H. S., Simpson, W. A., and Beachey, E. H. (1986) Relationship of critical micelle concentrations of bacterial lipoteichoic acids to biological activities. Infect Immun 51, 414-418 
30. Waldow, F., Kohler, T. P., Heß, N., Schwudke, D., Hammerschmidt, S., and Gisch, N. (2018) Attachment of phosphorylcholine residues to pneumococcal teichoic acids and modification of substitution patterns by the phosphorylcholine esterase. J Biol Chem 293, 10620-10629

31. Denapaite, D., Rieger, M., Köndgen, S., Brückner, R., Ochigava, I., Kappeler, P., MätzRensing, K., Leendertz, F., and Hakenbeck, R. (2016) Highly variable Streptococcus oralis strains are common among viridans Streptococci isolated from primates. mSphere 1, e00041-00015

32. Garriss, G., Nannapaneni, P., Simões, A. S., Browall, S., Subramanian, K., Sá-Leão, R., Goossens, H., de Lencastre, H., and Henriques-Normark, B. (2019) Genomic characterization of the emerging pathogen Streptococcus pseudopneumoniae. MBio 10

33. Martin, B., Humbert, O., Camara, M., Guenzi, E., Walker, J., Mitchell, T., Andrew, P., Prudhomme, M., Alloing, G., Hakenbeck, R., Morrison, D. A., Boulnois, G. J., and Claverys, J.-P. (1992) A highly conserved repeated DNA element located in the chromosome of Streptococcus pneumoniae. Nucleic Acids Res 20, 3479-3483

34. Knutsen, E., Johnsborg, O., Quentin, Y., Claverys, J.-P., and Håvarstein, L. S. (2006) BOX elements modulate gene expression in Streptococcus pneumoniae: impact on the fine-tuning of competence development. J Bacteriol 188, 8307-8312

35. Villegas, A., and Kropinski, A. M. (2008) An analysis of initiation codon utilization in the Domain Bacteria - concerns about the quality of bacterial genome annotation. Microbiology (Reading) 154, 2559-2661

36. Sabelnikov, A. G., Greenberg, B., and Lacks, S. A. (1995) An extended -10 promoter alone directs transcription of the DpnII operon of Streptococcus pneumoniae. J Mol Biol 250, 144-155

37. Vialle, S., Sepulcri, P., Dubayle, J., and Talaga, P. (2005) The teichoic acid (Cpolysaccharide) synthesized by Streptococcus pneumoniae serotype 5 has a specific structure. Carbohydr Res 340, 91-96

38. Meiers, M., Volz, C., Eisel, J., Maurer, P., Henrich, B., and Hakenbeck, R. (2014) Altered lipid composition in Streptococcus pneumoniae cpoA mutants. BMC Microbiol 14, 12

39. Hogg, S. D., Whiley, R. A., and De Soet, J. J. (1997) Occurrence of lipoteichoic acid in oral streptococci. Int J Syst Bacteriol 47, 62-66

40. Wei, Y., Joyce, L. R., Wall, A. M., Guan, Z., and Palmer, K. L. (2021) Streptococcus pneumoniae, S. mitis, and S. oralis produce a phosphatidylglycerol-dependent, ltaSIndependent glycerophosphate-linked glycolipid. mSphere 6, e01099-01020

41. Di Guilmi, A. M., Bonnet, J., Peißert, S., Durmort, C., Gallet, B., Vernet, T., Gisch, N., and Wong, Y.-S. (2017) Specific and spatial labeling of choline-containing teichoic acids in Streptococcus pneumoniae by click chemistry. Chem Commun (Camb) 53, 10572-10575

42. Alloing, G., Granadel, C., Morrison, D. A., and Claverys, J.-P. (1996) Competence pheromone, oligopeptide permease, and induction of competence in Streptococcus pneumoniae. Mol Microbiol 21, 471-478

43. Pirson, C., Engel, R., Jones, G. J., Holder, T., Holst, O., and Vordermeier, H. M. (2015) Highly purified mycobacterial phosphatidylinositol mannosides drive cell-mediated responses and activate NKT cells in cattle. Clin Vaccine Immunol 22, 178-184

44. Gottlieb, H. E., Kotlyar, V., and Nudelman, A. (1997) NMR chemical shifts of common laboratory solvents as trace impurities. J Org Chem 62, 7512-7515

45. Johnson, M., Zaretskaya, I., Raytselis, Y., Merezhuk, Y., McGinnis, S., and Madden, T. L. (2008) NCBI BLAST: a better web interface. Nucleic Acids Res 36, W5-9 
46. Zuker, M. (2003) Mfold web server for nucleic acid folding and hybridization prediction. Nucleic Acids Res 31, 3406-3415

47. Letunic, I., Khedkar, S., and Bork, P. (2021) SMART: recent updates, new developments and status in 2020. Nucleic Acids Res 49, D458-d460

\section{ABBREVIATIONS}

Ala, alanine; DAG, diacyl-glycerol; Gal, galactose; Glc, glucose; Gro, glycerol; HMQC, heteronuclear multiple quantum correlation; HSQC, heteronuclear single quantum correlation; LTA, lipoteichoic acid; ppm, parts per million; RU, repeating unit; TOCSY, total correlation spectroscopy.

Table 1. ${ }^{1} \mathrm{H}(700.4 \mathrm{MHz}),{ }^{13} \mathrm{C}$ NMR (176.1 MHz), and ${ }^{31} \mathrm{P}$ NMR $(283.5 \mathrm{MHz})$ chemical shift data $(\delta, \mathrm{ppm})[J, \mathrm{~Hz}]$ for $S$. mitis strain B6 type I LTA after hydrazine treatment ( $O$-deacyl LTA) recorded in deuterated $25 \mathrm{mM}$ sodium phosphate buffer (pH 5.5) at $300 \mathrm{~K}$.

\begin{tabular}{|c|c|c|c|c|c|c|}
\hline Residue & H-1 & H-2 & H-3 & H-4 & H-5 & H-6 \\
\hline (assignment) & $C-1$ & $C-2$ & $C-3$ & $C-4$ & $C-5$ & $C-6$ \\
\hline Gro- $\left(1 \rightarrow\left[\right.\right.$ Gro $\left.^{\mathbf{L A I}}\right]$ & $\begin{array}{c}3.76-3.73^{*} \\
3.65-3.61^{*} \\
69.2\end{array}$ & $\begin{array}{c}3.97-3.93^{*} \\
\text { n.d. }\end{array}$ & $\begin{array}{c}3.70-3.65^{*} \\
3.62-3.58^{*} \\
63.2\end{array}$ & & & \\
\hline$\rightarrow 1)-\beta-$ D-Galf- $(6 \rightarrow P[$ Gal $f]$ & $\begin{array}{c}5.04-5.02^{*} \\
107.9\end{array}$ & $\begin{array}{c}4.11-4.09^{*} \\
81.6\end{array}$ & $\begin{array}{c}4.10-4.07 * \\
77.4\end{array}$ & $\begin{array}{c}4.05-4.01 * \\
83.5\end{array}$ & $\begin{array}{c}3.99-3.96^{*} \\
70.2\end{array}$ & $\begin{array}{l}3.96-3.92 * \\
67.1[5.3]\end{array}$ \\
\hline$P \rightarrow 1)$-Gro-(3 $\rightarrow P[$ Gro-P $]$ & $\begin{array}{c}4.00-3.95^{*} \\
3.93-3.87^{*} \\
66.9[6.4]\end{array}$ & $\begin{array}{c}4.08-4.03 * \\
70.1\end{array}$ & $\begin{array}{r}4.00-3.95 * \\
3.93-3.87 * \\
66.9[6.4]\end{array}$ & & & \\
\hline$P \rightarrow 1)$-Gro $\left[\mathbf{G r o}^{\text {term }}\right]$ & $\begin{array}{c}3.95-3.91^{*} \\
3.89-3.85^{*} \\
67.0[5.2]\end{array}$ & $\begin{array}{c}3.93-3.90^{*} \\
70.9\end{array}$ & $\begin{array}{c}3.70-3.66 * \\
3.63-3.59 * \\
62.7\end{array}$ & & & \\
\hline${ }^{31} \mathbf{P}^{\#}$ & $\mathrm{Gal}_{\mathrm{f}}-6-P-\mathrm{Gr}$ & .d.; Gro- $P-\mathrm{G}$ & $1.48-1.12$. & & & \\
\hline
\end{tabular}


Table 2. ${ }^{1} \mathrm{H}(700.4 \mathrm{MHz}),{ }^{13} \mathrm{C}$ NMR (176.1 MHz), and ${ }^{31} \mathrm{P}$ NMR $(283.5 \mathrm{MHz})$ chemical shift data $(\delta$, ppm) $[J, \mathrm{~Hz}]$ for $S$. mitis strain B6 type IV LTA after hydrazine treatment $(O$-deacyl LTA) recorded in deuterated $25 \mathrm{mM}$ sodium phosphate buffer (pH 5.5) at $300 \mathrm{~K}$.

\begin{tabular}{|c|c|c|c|c|c|c|c|}
\hline $\begin{array}{c}\text { Residue } \\
\text { (assignment) }\end{array}$ & $\begin{array}{l}\mathrm{H}-1 \\
\mathrm{C}-1\end{array}$ & $\begin{array}{l}\mathrm{H}-2 \\
\mathrm{C}-2 \\
\end{array}$ & $\begin{array}{l}\mathrm{H}-3 \\
\mathrm{C}-3 \\
\end{array}$ & $\begin{array}{l}\mathrm{H}-4 \\
\mathrm{C}-4 \\
\end{array}$ & $\begin{array}{l}\mathrm{H}-5 \\
\mathrm{C}-5 \\
\end{array}$ & $\begin{array}{l}\mathrm{H}-6 \\
\mathrm{C}-6\end{array}$ & $\overline{\text { NAc }}$ \\
\hline Gro- $\left(1 \rightarrow\left[\right.\right.$ Gro $\left.^{\text {LAIV }}\right]$ & $\begin{array}{c}3.83-3.79^{*} \\
3.50-3.46^{*} \\
\quad 69.5\end{array}$ & $\begin{array}{c}3.97-3.94 * \\
\text { n.d. }\end{array}$ & $\begin{array}{c}3.70-3.65^{*} \\
3.62-3.58^{*} \\
63.1\end{array}$ & & & & \\
\hline $\begin{array}{c}\rightarrow 3)-\alpha-\mathrm{D}-\mathrm{Glcp}-(1 \rightarrow \\
{[\mathbf{A}]}\end{array}$ & $\begin{array}{c}4.88[3.8] \\
99.4\end{array}$ & $\begin{array}{c}3.64-3.60 * \\
71.8\end{array}$ & $\begin{array}{c}3.89-3.85^{*} \\
82.3\end{array}$ & $\begin{array}{c}3.50-3.46^{*} \\
68.6\end{array}$ & $\begin{array}{c}3.71-3.67 * \\
72.2\end{array}$ & $\begin{array}{l}3.86-3.83 * \\
3.77-3.74 * \\
\quad 61.1\end{array}$ & \\
\hline $\begin{array}{l}\rightarrow 3)-\beta \text {-AATGal } p- \\
(1 \rightarrow(\mathbf{B})\end{array}$ & $\begin{array}{c}4.78-4.75 * \\
102.3\end{array}$ & $\begin{array}{l}3.93-3.88 * \\
51.8\end{array}$ & $\begin{array}{l}4.21-4.17^{*} \\
78.0\end{array}$ & $\begin{array}{l}3.94-3.91 * \\
55.1\end{array}$ & $\begin{array}{c}4.06-4.01 * \\
67.8\end{array}$ & $\begin{array}{c}1.36[6.7] \\
16.4\end{array}$ & $\begin{array}{l}2.03 \\
22.9 \\
175.8\end{array}$ \\
\hline $\begin{array}{l}P \rightarrow 6)-\beta-\mathrm{D}-\mathrm{Gal} p-(1 \rightarrow \\
\left(\mathbf{C}^{\prime}\right)\end{array}$ & $\begin{array}{c}4.63[7.9] \\
105.2\end{array}$ & $\begin{array}{l}3.57-3.53 * \\
\quad 71.0\end{array}$ & $\begin{array}{c}3.67-3.63 * \\
72.9\end{array}$ & $\begin{array}{l}3.99-3.95 * \\
68.8\end{array}$ & $\begin{array}{l}3.88-3.85^{*} \\
74.3-74.1\end{array}$ & $\begin{array}{c}4.05-3.99 * \\
65.3-65.1\end{array}$ & \\
\hline $\begin{array}{l}\rightarrow 1) \text {-ribitol-(5 } \rightarrow P \\
(\text { Rib-ol- } P)\end{array}$ & $\begin{array}{c}3.97-3.93^{*} \\
3.88-3.83^{*} \\
\quad 71.1\end{array}$ & $\begin{array}{c}4.01-3.96^{*} \\
71.0\end{array}$ & $\begin{array}{c}3.78-3.74 * \\
72.1\end{array}$ & $\begin{array}{c}3.91-3.87 * \\
71.2\end{array}$ & $\begin{array}{l}4.06-4.02 * \\
3.99-3.94 * \\
67.2[5.3]\end{array}$ & & \\
\hline $\begin{array}{l}\rightarrow 3)-\beta-\mathrm{D}-6-O-P \text {-Cho- } \\
\text { Gal } p \text { NAc }(1 \rightarrow(\mathbf{D})\end{array}$ & $\begin{array}{c}4.63-4.59 * \\
101.9\end{array}$ & $\begin{array}{l}4.12-4.07 * \\
51.3\end{array}$ & $\begin{array}{c}3.88-3.83^{*} \\
75.3\end{array}$ & $\begin{array}{l}4.19-4.15^{*} \\
63.9\end{array}$ & $\begin{array}{l}3.85-3.81 * \\
74.3-74.1\end{array}$ & $\begin{array}{l}4.09-4.04 * \\
65.3-65.1\end{array}$ & $\begin{array}{l}2.08 \\
23.1 \\
175.3\end{array}$ \\
\hline $\begin{array}{l}\rightarrow 4)-\alpha-\mathrm{D}-6-O-P-\mathrm{Cho}- \\
\text { Gal } p \text { NAc }(1 \rightarrow(\mathbf{E})\end{array}$ & $\begin{array}{l}5.16[3.2] \\
\quad 94.0\end{array}$ & $\begin{array}{c}4.34-4.29 * \\
50.0\end{array}$ & $\begin{array}{c}3.94-3.90^{*} \\
67.4\end{array}$ & $\begin{array}{c}4.11-4.09^{*} \\
77.2\end{array}$ & $\begin{array}{l}4.02-3.98 * \\
71.2\end{array}$ & $\begin{array}{l}4.04-3.96^{*} \\
64.3-64.1 *\end{array}$ & $\begin{array}{l}2.05 \\
22.7 \\
175.2\end{array}$ \\
\hline $\begin{array}{l}\rightarrow 3)-\alpha-\text { AATGal } p- \\
(1 \rightarrow(\mathbf{F})\end{array}$ & $\begin{array}{l}4.96[3.8] \\
98.8\end{array}$ & $\begin{array}{c}4.23-4.19^{*} \\
48.9\end{array}$ & $\begin{array}{c}4.43-4.39^{*} \\
75.2\end{array}$ & $\begin{array}{l}3.99-3.96^{*} \\
55.4\end{array}$ & $\begin{array}{l}4.80-4.76^{*} \\
\quad 63.6\end{array}$ & $\begin{array}{l}1.25[6.7] \\
16.2\end{array}$ & $\begin{array}{l}2.10 \\
22.7 \\
175.1\end{array}$ \\
\hline $\begin{array}{l}P \rightarrow 6)-\beta-\mathrm{D}-\mathrm{Gal} p-(1 \rightarrow \\
(\mathbf{C})\end{array}$ & $\begin{array}{l}4.62-4.58^{*} \\
105.0\end{array}$ & $\begin{array}{l}3.55-3.51 * \\
\quad 71.1\end{array}$ & $\begin{array}{c}3.69-3.65^{*} \\
73.0\end{array}$ & $\begin{array}{c}3.99-3.96^{*} \\
68.8\end{array}$ & $\begin{array}{c}3.88-3.85^{*} \\
74.3-74.1\end{array}$ & $\begin{array}{c}4.05-3.99 * \\
65.3-65.1\end{array}$ & \\
\hline $\begin{array}{l}\rightarrow 3)-\beta \text {-D-6-O-P-Cho- } \\
\text { Gal } p \text { NAc }\left(1 \rightarrow\left(\mathbf{D}^{\text {term }}\right)\right.\end{array}$ & $\begin{array}{l}4.59-4.57^{*} \\
101.9\end{array}$ & $\begin{array}{l}4.12-4.07 * \\
51.2\end{array}$ & $\begin{array}{l}3.85-3.81 * \\
75.3\end{array}$ & $\begin{array}{c}4.16-4.13^{*} \\
63.9\end{array}$ & $\begin{array}{l}3.85-3.81 * \\
74.3-74.1\end{array}$ & $\begin{array}{l}4.09-4.03 * \\
65.3-65.1\end{array}$ & $\begin{array}{l}2.07 \\
22.8 \\
175.3\end{array}$ \\
\hline $\begin{array}{l}\rightarrow 4)-\alpha-D-6-O-P-C h o- \\
\text { Gal } p \text { NAc }\left(\mathbf{E}^{\text {term }}\right)\end{array}$ & $5.08[3.3]$ & $4.24-4.20 *$ & $3.82-3.77 *$ & $4.05-4.01 *$ & n.d. & $\begin{array}{l}4.07-4.04 * \\
4.03-3.98^{*}\end{array}$ & 2.04 \\
\hline & 94.1 & 50.0 & 68.2 & 68.6 & n.d. & $65.3-65.1$ & $\begin{array}{l}22.7 \\
175.2\end{array}$ \\
\hline $\begin{array}{l}\text { Cho- } P-(6-O \rightarrow \\
@ \mathbf{D}, \mathbf{D}^{\text {term }}, \mathbf{E}^{\text {term }}\end{array}$ & $\begin{array}{l}4.36-4.31^{*} \\
60.2[5.2]\end{array}$ & $\begin{array}{c}3.71-3.67 * \\
66.6\end{array}$ & $\begin{array}{l}3.23 \\
54.6\end{array}$ & & & & \\
\hline $\begin{array}{l}\text { Cho-P-(6-O } \rightarrow \\
@ \mathbf{E}\end{array}$ & $\begin{array}{l}4.30-4.26^{*} \\
60.1[5.1]\end{array}$ & $\begin{array}{l}3.69-3.64 * \\
66.6\end{array}$ & $\begin{array}{l}3.23 \\
54.6\end{array}$ & & & & \\
\hline${ }^{31} \mathbf{P}^{\#}$ & \multicolumn{7}{|c|}{ 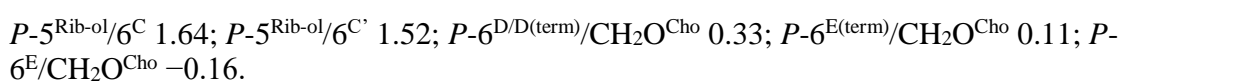 } \\
\hline
\end{tabular}

${ }^{\#}$ shifts from spectra recorded in $\mathrm{D}_{2} \mathrm{O} @ 300 \mathrm{~K}$ (see Fig. 2); *non-resolved multiplet; n.d. = not detected. 
Table 3. Mass spectrometric analysis of $O$-deacyl type I LTA of $S$. mitis strains B6 and NCTC10712. Summary of calculated monoisotopic neutral masses and observed molecular masses [Da] for LTA preparations after hydrazine treatment. For each preparation, independent MS analyses have been performed and identified molecules are listed as a combined list. Masses observed only in one of the two preparations of a strain are written in italic style. Accuracy of the measurement is stated as $\Delta \mathrm{ppm}$.

\begin{tabular}{|c|c|c|c|c|c|}
\hline \multirow[b]{2}{*}{ Molecule } & \multirow[b]{2}{*}{$\begin{array}{l}\text { calculated } \\
\text { mass [Da] }\end{array}$} & \multicolumn{2}{|c|}{ Strain B6 } & \multicolumn{2}{|c|}{ Strain NCTC10712 } \\
\hline & & $\begin{array}{l}\text { observed } \\
\text { mass [Da] }\end{array}$ & $\begin{array}{c}\text { accuracy } \\
{[\Delta \mathrm{ppm}]}\end{array}$ & $\begin{array}{l}\text { observed } \\
\text { mass [Da] }\end{array}$ & $\begin{array}{r}\text { accuracy } \\
{[\Delta \mathrm{ppm}]}\end{array}$ \\
\hline$\left(\right.$ GroP) ${ }_{4}$ GalfGro & 870.113 & 870.115 & 2.3 & 870.114 & 1.2 \\
\hline$(\text { GroP) })_{5}$ GalfGro & 1024.116 & 1024.117 & 1.0 & 1024.117 & 1.0 \\
\hline (GroP)6GalfGro & 1178.119 & 1178.120 & 0.8 & 1178.120 & 0.8 \\
\hline$(\text { GroP) })_{7}$ GalfGro & 1332.122 & 1332.124 & 1.5 & 1332.123 & 0.8 \\
\hline$(\text { GroP) })_{8}$ GalfGro & 1486.125 & 1486.128 & 2.0 & 1486.127 & 1.3 \\
\hline$\left(\right.$ GroP) ${ }_{9}$ GalfGro & 1640.128 & 1640.132 & 2.4 & 1640.131 & 1.8 \\
\hline$(\text { GroP })_{10}$ GalfGro & 1794.131 & 1794.135 & 2.2 & 1794.134 & 1.7 \\
\hline$(\text { GroP })_{11}$ GalfGro & 1948.134 & 1948.139 & 2.6 & 1948.138 & 2.1 \\
\hline$(\text { GroP })_{12}$ GalfGro & 2102.138 & 2102.142 & 1.9 & 2102.140 & 1.0 \\
\hline$(\text { GroP })_{13}$ GalfGro & 2256.141 & 2256.145 & 1.8 & 2256.144 & 1.3 \\
\hline$(\text { GroP })_{14}$ GalfGro & 2410.144 & 2410.148 & 1.7 & 2410.147 & 1.2 \\
\hline$(\text { GroP })_{15}$ GalfGro & 2564.147 & 2564.151 & 1.6 & 2564.150 & 1.2 \\
\hline$(\text { GroP })_{16}$ GalfGro & 2718.150 & 2718.155 & 1.8 & 2718.154 & 1.5 \\
\hline$(\text { GroP })_{17}$ Gal $f$ Gro & 2872.153 & 2872.158 & 1.8 & 2872.157 & 1.4 \\
\hline
\end{tabular}


Table 4. Mass spectrometric analysis of $O$-deacyl type IV LTA of $S$. mitis strains B6 and NCTC10712. Summary of calculated monoisotopic neutral masses and observed molecular masses [Da] for LTA preparations after hydrazine treatment. For each preparation, independent MS analyses have been performed. Identified molecules from one analysis per strain (spectra shown in Fig. 4) are listed. Masses observed only in very minor intensity are written in italic style. Accuracy of the measurement is stated as $\Delta \mathrm{ppm} ;$ n.d. $=$ not detected; ${ }^{\#}=1^{\text {st }}$ isotopic peak, ${ }^{\S}=2^{\text {nd }}$ isotopic peak.

\begin{tabular}{|c|c|c|c|c|c|}
\hline \multirow[b]{2}{*}{ Molecule } & \multirow[b]{2}{*}{$\begin{array}{l}\text { calculated } \\
\text { mass [Da] }\end{array}$} & \multicolumn{2}{|c|}{ Strain B6 } & \multicolumn{2}{|c|}{ Strain NCTC10712 } \\
\hline & & $\begin{array}{c}\text { observed } \\
\text { mass [Da] }\end{array}$ & $\begin{array}{c}\text { accuracy } \\
{[\Delta \mathrm{ppm}]}\end{array}$ & $\begin{array}{l}\text { observed } \\
\text { mass [Da] }\end{array}$ & $\begin{array}{c}\text { accuracy } \\
{[\Delta \mathrm{ppm}]}\end{array}$ \\
\hline $4 \mathrm{RU}$ & 5447.89 & 5447.90 & 1.8 & $5448.90^{\#}$ & 1.8 \\
\hline - 1 P-Cho & 5282.83 & 5282.85 & 3.8 & 5282.84 & 1.9 \\
\hline - 2 P-Cho & 5117.78 & n.d. & - & n.d. & - \\
\hline 5 RU & 6746.34 & 6746.33 & -1.5 & 6746.36 & 3.0 \\
\hline - 1 P-Cho & 6581.28 & 6581.29 & 1.5 & 6581.29 & 1.5 \\
\hline - 2 P-Cho & 6416.23 & 6416.22 & -1.6 & 6416.24 & 1.6 \\
\hline 6 RU & 8044.78 & 8044.78 & 0.0 & $8045.78^{\#}$ & -1.2 \\
\hline - 1 P-Cho & 7879.73 & 7879.72 & -1.3 & 7879.72 & -1.3 \\
\hline - 2 P-Cho & 7714.67 & 7714.67 & 0.0 & 7714.67 & 0.0 \\
\hline $7 \mathrm{RU}$ & 9343.23 & 9343.20 & -3.2 & 9343.13 & -10.7 \\
\hline - 1 P-Cho & 9178.18 & 9178.15 & -3.3 & 9178.15 & -3.3 \\
\hline - 2 P-Cho & 9013.12 & 9013.11 & -1.1 & 9013.10 & -2.2 \\
\hline 8 RU & 10641.68 & n.d. & - & n.d. & - \\
\hline - 1 P-Cho & 10476.62 & n.d. & - & $10477.58^{\#}$ & -4.8 \\
\hline - 2 P-Cho & 10311.57 & $10313.55^{\S}$ & 1.9 & $10313.57^{\S}$ & 0.0 \\
\hline
\end{tabular}


Table 5. ${ }^{1} \mathrm{H}(700.4 \mathrm{MHz})$ and ${ }^{13} \mathrm{C}$ NMR (176.1 MHz) chemical shift data $(\delta, \mathrm{ppm})[J, \mathrm{~Hz}]$ for the carbohydrate moieties of glycosylglycerolipids isolated from $S$. mitis strain $\mathrm{B} 6$ recorded in $\mathrm{MeOH}$ $d_{4}$ at $300 \mathrm{~K}$. *non-resolved multiplet.

\begin{tabular}{|c|c|c|c|c|c|c|}
\hline & \multicolumn{2}{|c|}{$\beta$-Gal $f$-(1,3)-DAG } & \multicolumn{2}{|c|}{$\alpha$-Glcp-(1,3)-DAG } & \multicolumn{2}{|c|}{$\alpha$-Gal $p-(1,2)-\alpha-G l c p$-(1,3)-DAG } \\
\hline & $\delta \mathrm{H} / \delta_{C}[\mathrm{ppm}]$ & $J[\mathrm{~Hz}]$ & $\delta_{\mathrm{H}} / \delta_{C}[\mathrm{ppm}]$ & $J[\mathrm{~Hz}]$ & $\delta \mathrm{H} / \delta_{C}[\mathrm{ppm}]$ & $J[\mathrm{~Hz}]$ \\
\hline & $\beta$-Gal $f-(1 \rightarrow$ & & $\alpha$-Glcp-(1) & & $\alpha$-Galp-(1) & \\
\hline $\mathrm{H}-1$ & $4.88(d)$ & 1.6 & 4.80 (d) & 3.7 & $5.02(\mathrm{~d})$ & 3.7 \\
\hline$C-1$ & 109.7 & & 100.7 & & 98.2 & \\
\hline $\mathrm{H}-2$ & $3.96(\mathrm{dd})$ & $3.9,1.6$ & $3.39(\mathrm{dd})$ & $9.7,3.7$ & $3.79-3.75^{*}$ & \\
\hline$C-2$ & 83.3 & & 73.5 & & 70.3 & \\
\hline H-3 & $4.01(\mathrm{dd})$ & $6.4,3.9$ & $3.65-3.60 *$ & & $3.83-3.79 *$ & \\
\hline$C-3$ & 78.7 & & 75.0 & & 71.4 & \\
\hline H-4 & $3.94(\mathrm{dd})$ & $6.4,3.4$ & $3.32-3.28 *$ & & $3.91-3.89 *$ & \\
\hline$C-4$ & 84.6 & & 71.7 & & 71.1 & \\
\hline H-5 & $3.74-3.70^{*}$ & & $3.60-3.55 *$ & & $4.12-4.07 *$ & \\
\hline$C-5$ & 72.4 & & 74.0 & & 72.6 & \\
\hline H-6a & $3.64-3.61 *$ & & $3.68(\mathrm{dd})$ & $11.8,5.4$ & $3.71-3.67 *$ & \\
\hline$C-6$ & 64.5 & & 62.6 & & 62.9 & \\
\hline H-6b & $3.64-3.61^{*}$ & & 3.79 (dd) & $11.8,2.4$ & $3.75-3.71^{*}$ & \\
\hline & & & & & $\rightarrow 2)-\alpha$-Glcp-(1 $\rightarrow$ & \\
\hline $\mathrm{H}-1$ & & & & & 5.05 (d) & 3.5 \\
\hline$C-1$ & & & & & 97.7 & \\
\hline $\mathrm{H}-2$ & & & & & $3.58(\mathrm{dd})$ & $9.7,3.5$ \\
\hline$C-2$ & & & & & 77.7 & \\
\hline $\mathrm{H}-3$ & & & & & $3.78-3.74 *$ & \\
\hline$C-3$ & & & & & 73.4 & \\
\hline $\mathrm{H}-4$ & & & & & $3.36(\mathrm{dd})$ & $9.5,9.3$ \\
\hline$C-4$ & & & & & 71.4 & \\
\hline H-5 & & & & & $3.61-3.57 *$ & \\
\hline$C-5$ & & & & & 73.9 & \\
\hline H-6a & & & & & $3.70-3.66 *$ & \\
\hline$C-6$ & & & & & 62.6 & \\
\hline H-6b & & & & & 3.82-3.79* & \\
\hline $\begin{array}{l}\rightarrow \text { 3)-Gro-(1,2- } \\
\text { diacyl) }\end{array}$ & & & & & & \\
\hline $\mathrm{H}-1 \mathrm{a}$ & $4.18(\mathrm{dd})$ & $12.1,6.8$ & $4.20(\mathrm{dd})$ & $12.0,6.5$ & $4.23(\mathrm{dd})$ & $12.0,6.6$ \\
\hline$C-1$ & 64.0 & & 63.8 & & 63.9 & \\
\hline $\mathrm{H}-1 \mathrm{~b}$ & $4.41(\mathrm{dd})$ & $12.1,2.9$ & 4.48 (dd) & $12.0,2.9$ & 4.48 (dd) & $12.0,2.6$ \\
\hline $\mathrm{H}-2$ & $5.26-5.21 *$ & & $5.28-5.23^{*}$ & & $5.27-5.22 *$ & \\
\hline$C-2$ & 71.6 & & 71.6 & & 71.5 & \\
\hline H-3a & $3.64-3.61 *$ & & $3.66-3.62(\mathrm{~m})$ & & $3.67(\mathrm{dd})$ & $10.9,5.4$ \\
\hline$C-3$ & 66.9 & & 67.1 & & 67.1 & \\
\hline$H-3 b$ & $3.82-3.79 *$ & & $3.87(\mathrm{dd})$ & $10.8,5.5$ & $3.88(\mathrm{dd})$ & $10.9,5.2$ \\
\hline
\end{tabular}




\section{Figure 1}

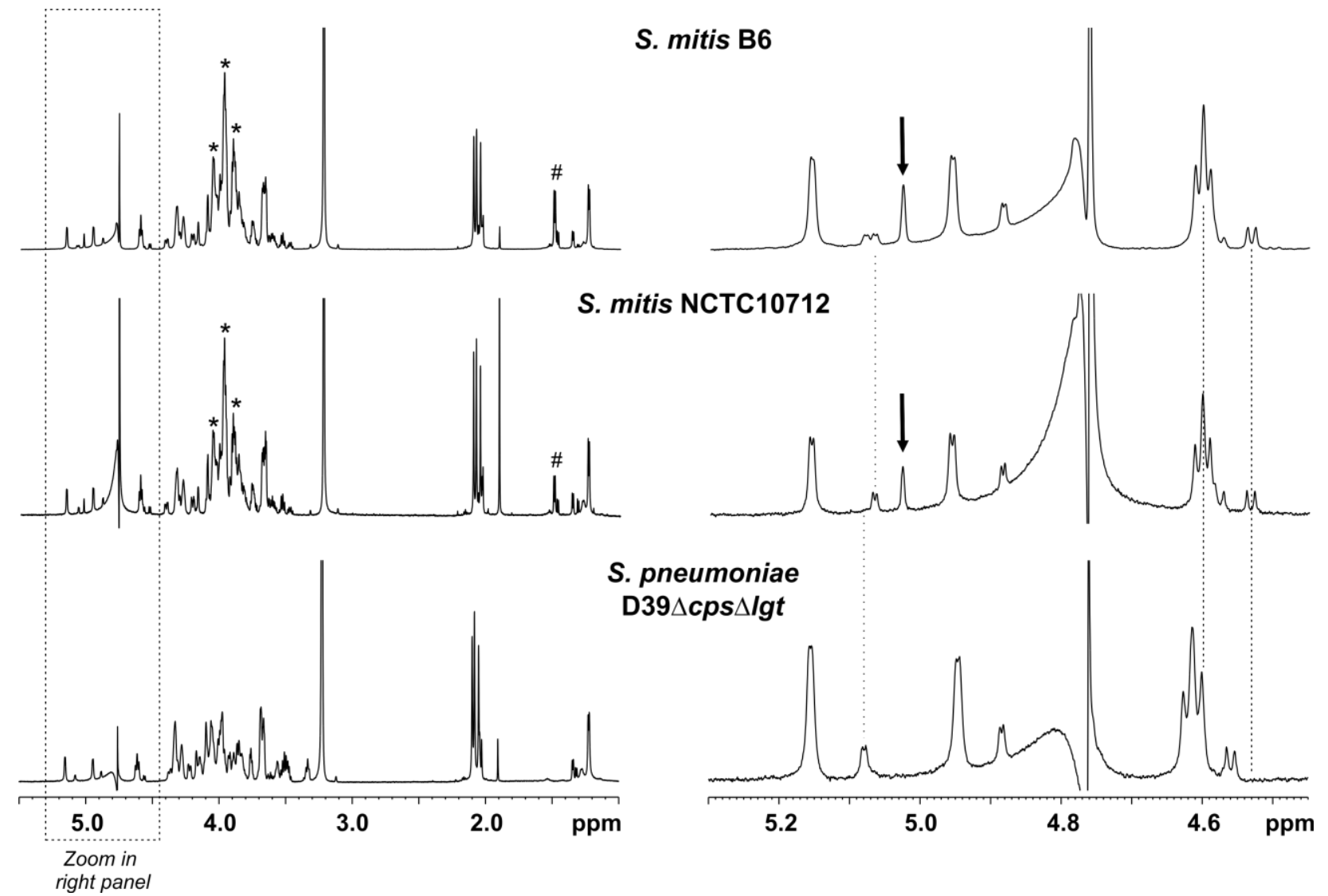

Figure 1. Identification of two LTA polymers in $S$. mitis strains. Shown are ${ }^{1} \mathrm{H}$ NMR spectra $\left(\delta_{\mathrm{H}}\right.$ 5.5-1.0 in left panel; ( $\delta_{\mathrm{H}} 5.3-4.45$ (anomeric region) in right panel) of $O$-deacylated LTA of $S$. mitis strains B6 (top) and NCTC10712 (middle) as well as from S. pneumoniae D39 $\Delta$ cps $\Delta l$ lgt (bottom), all recorded in deuterated $25 \mathrm{mM}$ sodium phosphate buffer $(\mathrm{pH}$ 5.5) at $300 \mathrm{~K}$. Dotted lines in right panel indicate deviating anomeric signals in the different LTA preparations. Stars in left panel as well as black arrows in right panel indicate the presence of an additional polymer in the $S$. mitis LTA preparations, which is absent in S. pneumoniae. Signals marked with \# (right panel) present in S. mitis LTA preparations result from a molecule that is formed during the hydrazine-mediated alanine-cleavage (most likely alanine-hydrazide), which was removed in the $S$. pneumoniae sample by chromatography (18). 


\section{Figure 2}

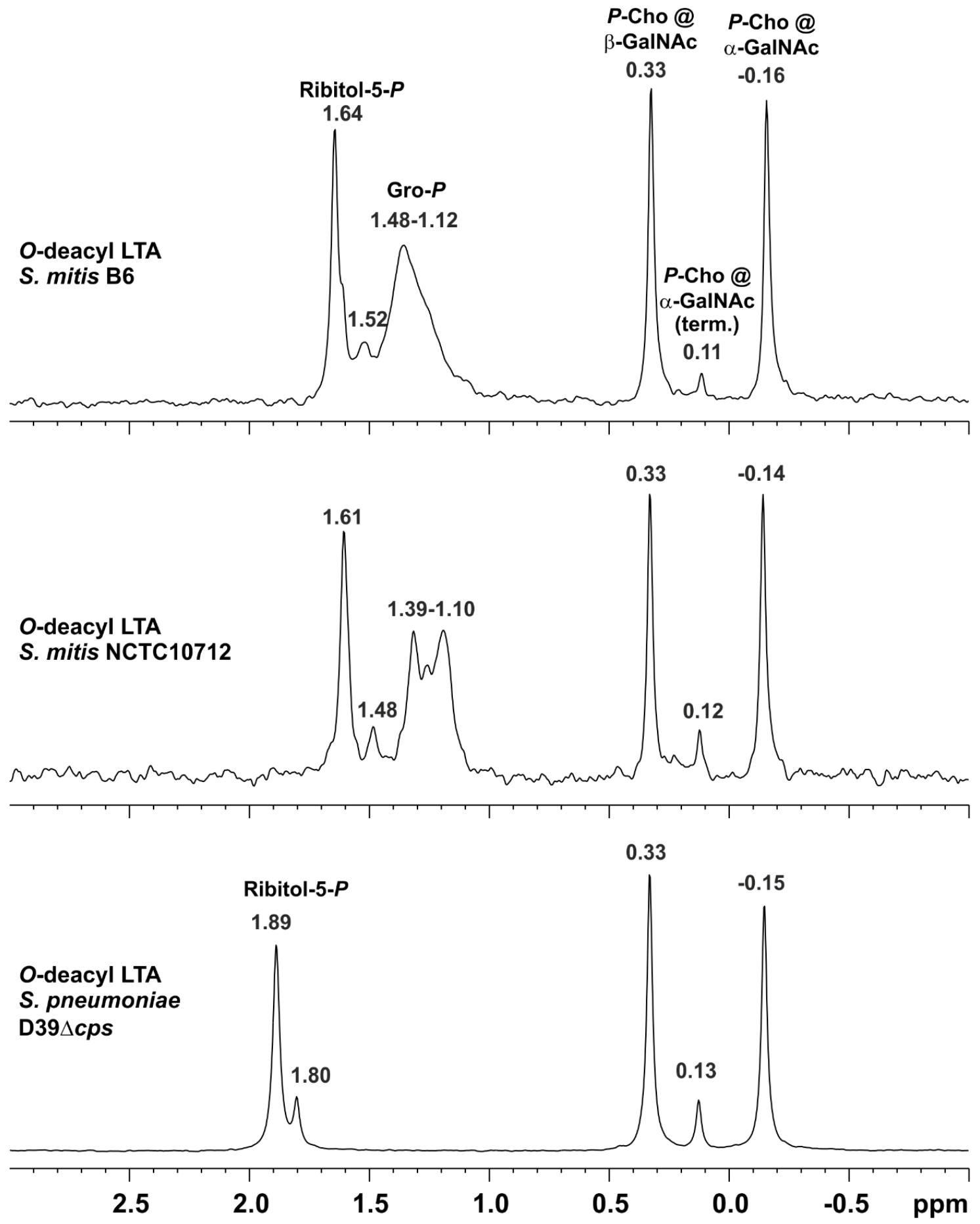

Figure 2. Presence of a poly-glycerolphosphate containing LTA in $\boldsymbol{S}$. mitis. Shown are ${ }^{31} \mathrm{P}$ NMR spectra $\left(\delta_{\mathrm{P}} 3.0-(-1.0)\right)$ of $O$-deacylated LTA of $S$. mitis strains B6 (top) and NCTC10712 (middle) as well as from $S$. pneumoniae D39 4 cps (bottom), recorded in $\mathrm{D}_{2} \mathrm{O}$ at $300 \mathrm{~K}$. The broad signal between $\delta_{\mathrm{P}} 1.48$ and $1.12 \mathrm{ppm}$ (B6) or 1.39 and $1.10 \mathrm{ppm}$ (NCTC10712), respectively, only present in $O$ deacylated LTA from $S$. mitis, clearly points to the presence of poly-glycerol-phosphate chains (compare (15)). 


\section{Figure 3}

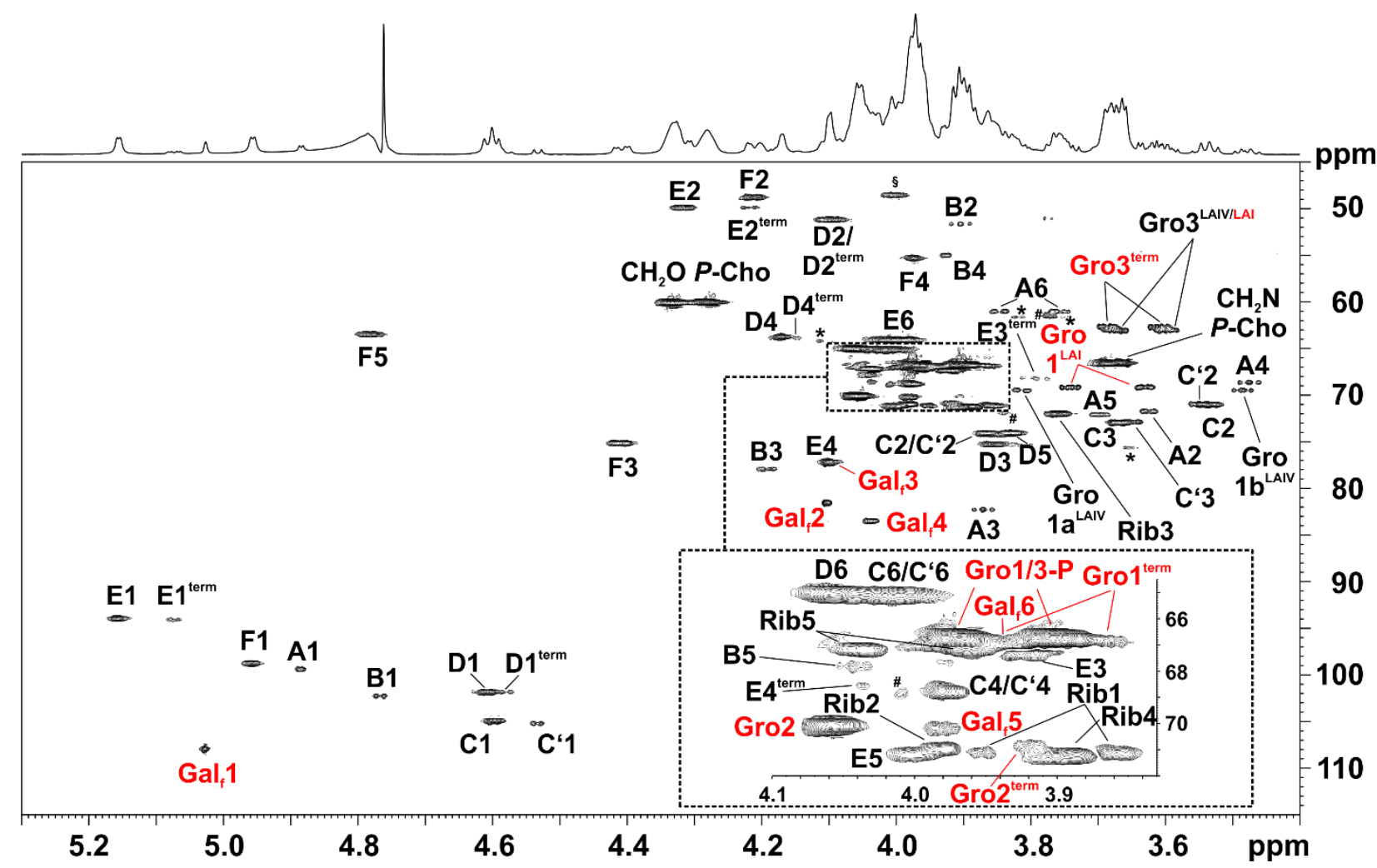

Figure 3. NMR analysis of $\boldsymbol{O}$-deacylated LTA of $\boldsymbol{S}$. mitis strain B6. Shown is a section $\left(\delta_{\mathrm{H}} 5.30\right.$ $3.40 ; \delta_{\mathrm{C}} 115-45$ ) of the ${ }^{1} \mathrm{H},{ }^{13} \mathrm{C}-\mathrm{HSQC}$ NMR spectrum (recorded in deuterated $25 \mathrm{mM}$ sodium phosphate buffer ( $\mathrm{pH} 5.5$ ) as dept-version) obtained from $O$-deacylated LTA of $S$. mitis strain B6 including assignment of signals (red: type I LTA; black: type IV LTA). The corresponding NMR chemical shift data are listed in Table 1 (type I LTA) and Table 2 (type IV LTA), respectively. 


\section{Figure 4}

\section{A) Type I LTA}
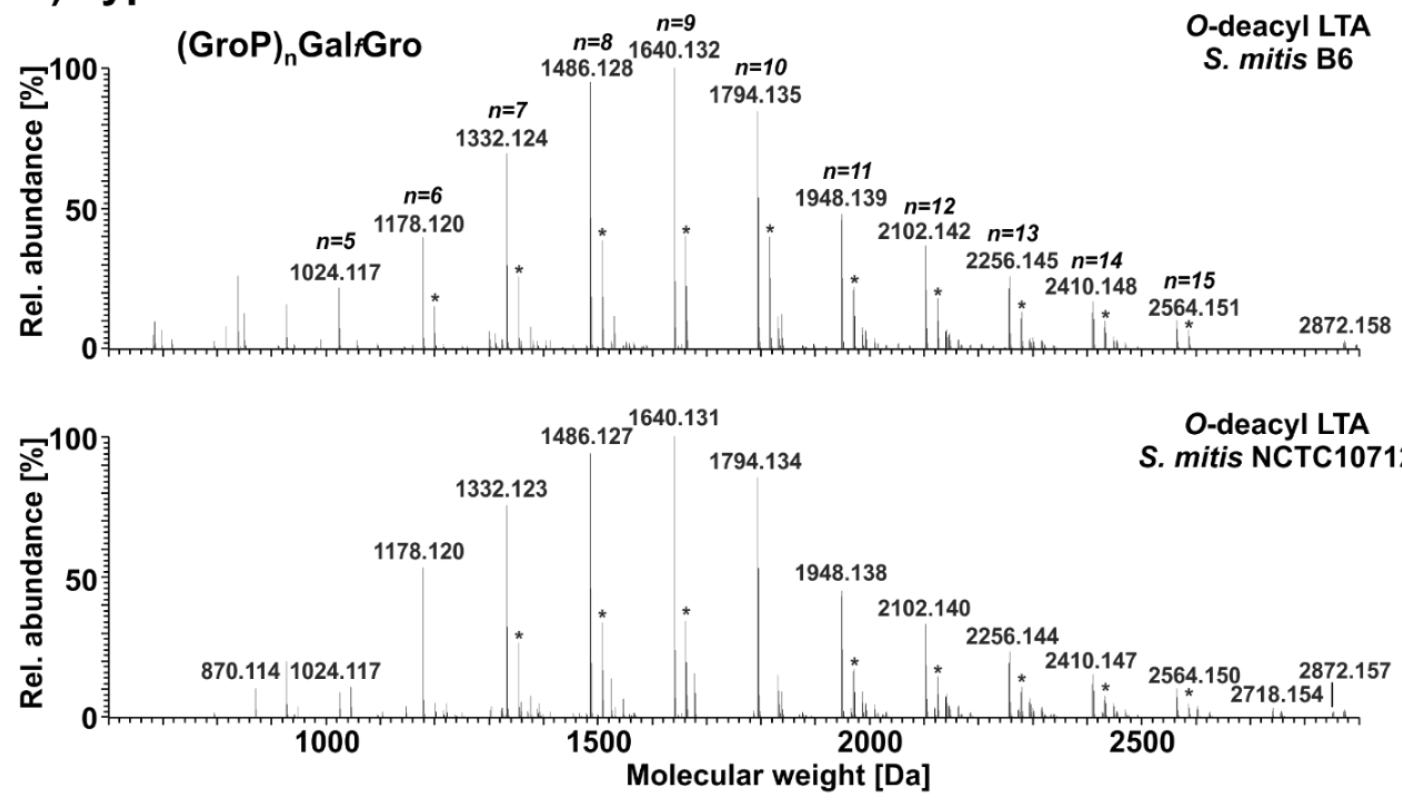

\section{B) Type IV LTA}

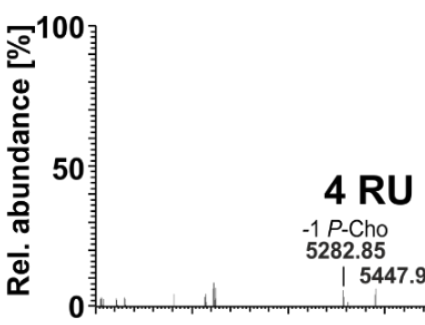

$\underset{-1 P-C h O}{6}$ -1 P-Cho
7879.73 7879.73

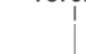
$-2 P$-Cho
7714.67 \begin{tabular}{l|l}
$\mathbf{7 7 1 4} .67$ & $\mathbf{8 0 4 4 . 7 8}$
\end{tabular}

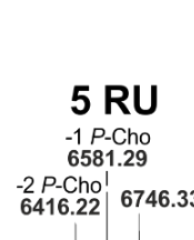

O-deacyl LTA

S. mitis B6

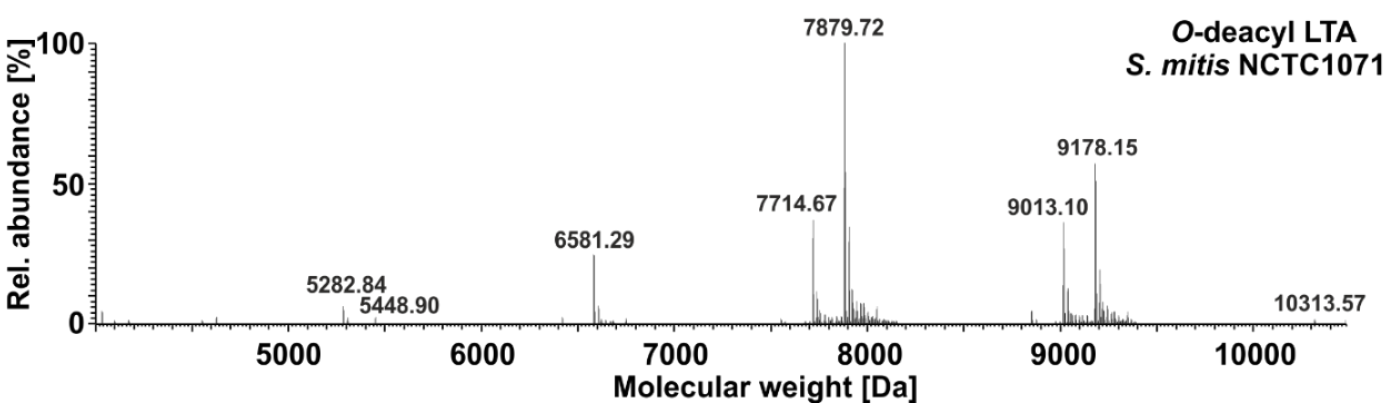

Figure 4. Molecular species distribution of $O$-deacylated LTA of $S$. mitis strains NCTC10712 and B6. A) Charge-deconvoluted spectra of a representative MS-analysis for each strain performed in the negative ion mode recorded in an $\mathrm{m} / z$-window of 500 to 1650 , focusing on the ionization of the type I LTA. Signals marked with * represent major abundant sodium adducts $(\Delta m=+21.98 \mathrm{Da}) . \mathrm{n}=$ number of GroP repeats. B) Charge-deconvoluted spectra of a representative MS-analysis for each strain performed in the negative ion mode recorded in an $\mathrm{m} / z$-window of 1600 to 3000 , focusing on the ionization of the type IV LTA. Details of all assigned molecular species are summarized in Table 3 (type I LTA) and Table 4 (type IV LTA), respectively. Relative abundance for a spectral region was always normalized to the respective base peak. 


\section{Figure 5}

LTA of S. mitis NCTC10712 and B6

Type I LTA

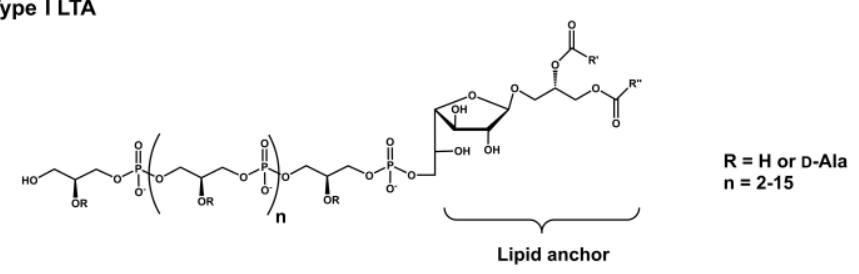

Type IV LTA

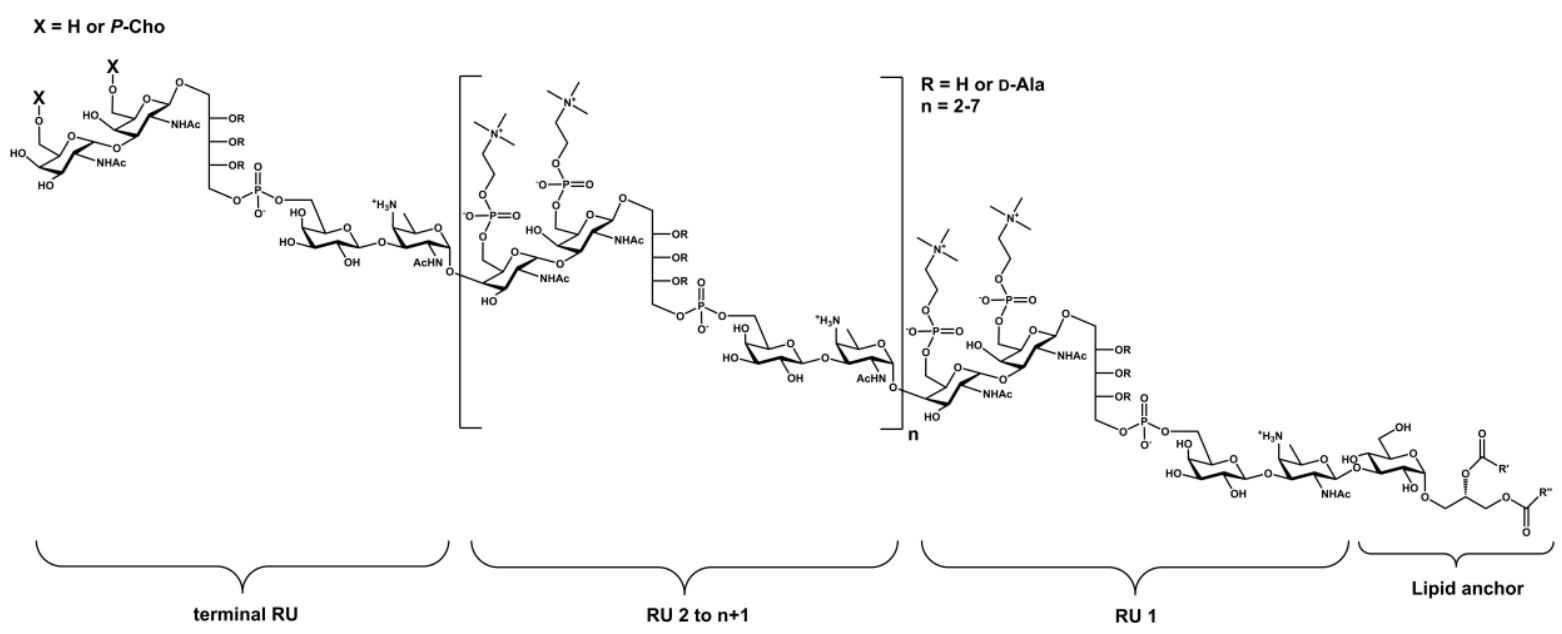

Figure 5. Chemical structures of LTA isolated from $S$. mitis strains NCTC10712 and B6. 


\section{Figure 6}

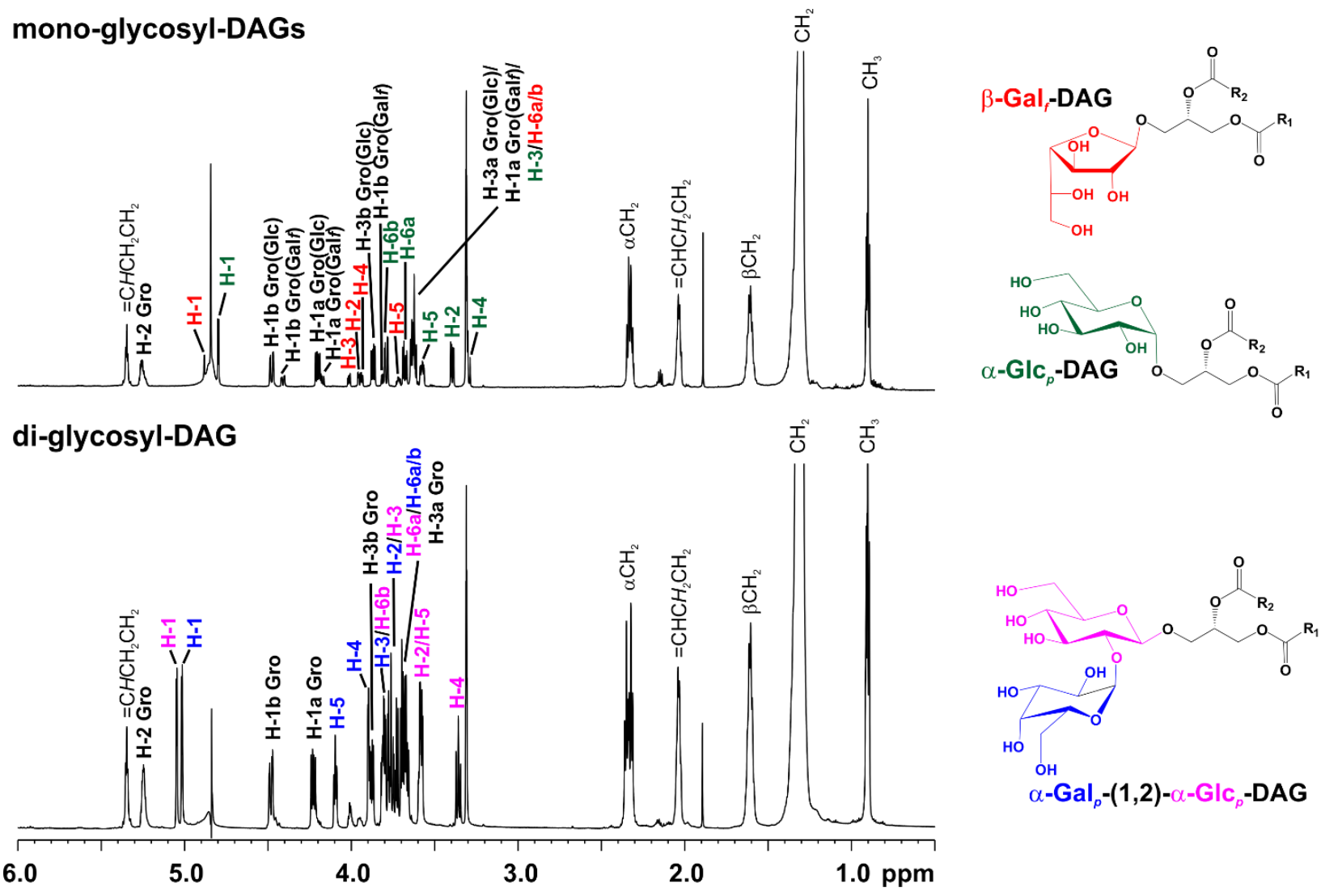

Figure 6. ${ }^{1} \mathrm{H}$ NMR analysis of glycosylglycerolipids isolated from $\boldsymbol{S}$. mitis strain B6. Shown are ${ }^{1} \mathrm{H}$ NMR spectra $\left(\delta_{\mathrm{H}} 6.0-0.5\right)$ of glycosylglycerolipids (top: mixture of two mono-glycosyl-diacylglycerols; bottom: one di-glycosyl-diacylglycerol) isolated from $S$. mitis strain B6, recorded in $\mathrm{MeOH}-d_{4}$ at 300 $\mathrm{K}$. The corresponding NMR chemical shift data are listed in Table 5, the specific chemical structures of the observed glycosylglycerolipids are depicted right hand in this figure. 


\section{Figure 7}

S. pneumoniae R6

S. pneumoniae D39

S. pneumoniae TIGR4

S. pneumoniae 70585

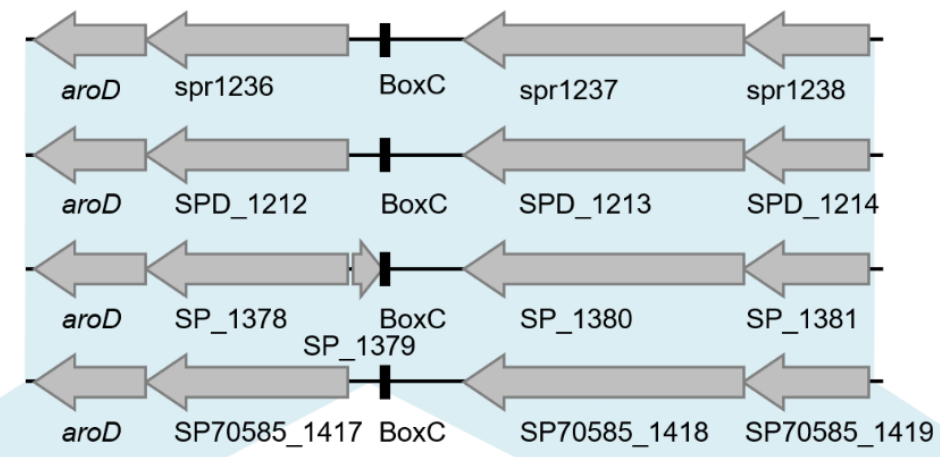

S. mitis $\mathrm{B} 6$

S. mitis NCTC10712
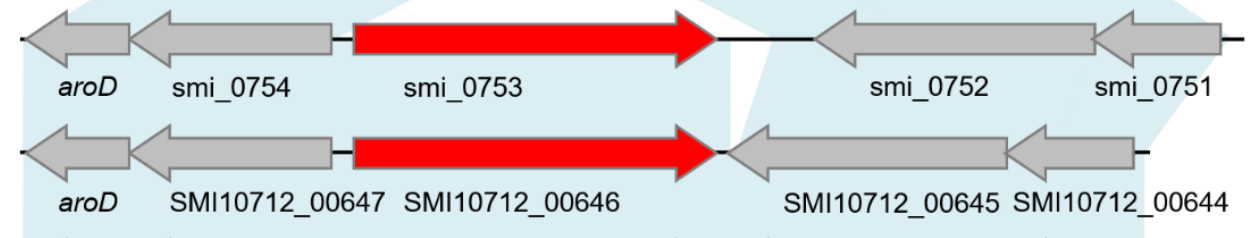

S. mitis NCTC12261

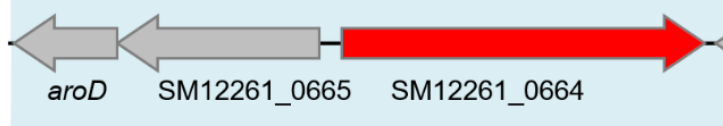

S. mitis SK137

S. pseudop. IS7493

S. pseudop.

ATCC BAA-960

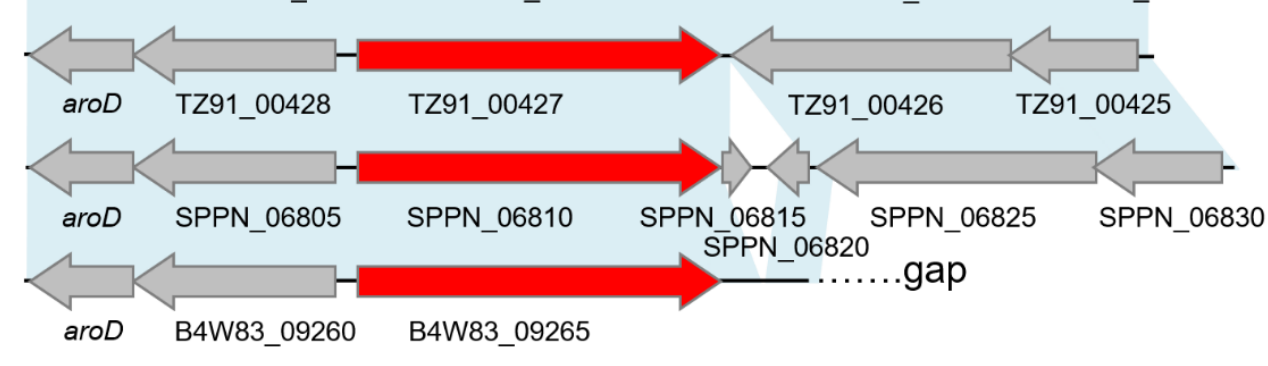

$500 \mathrm{bp}$

Figure 7. Comparison of the ltaS loci in three Streptococcus species. Representative strains of $S$. mitis and S. pseudopneumoniae carry an ltaS homolog (red). Upstream and downstream regions of the ltaS gene are conserved in all three species (genes are shown in grey and the old Gene/Locus nomenclature (sp/spr/smi) was used). Light blue areas illustrate BLASTn matches between sequences (90-93\% identity). Large arrows show the gene orientation and are drawn in scale as indicated by the $500 \mathrm{bp}$ bar. BoxC elements annotated in S. pneumoniae genomes are shown in black. The downstream region of the ltaS gene in the type strain of S. pseudopneumoniae ATCC BAA_960 is located in a gap. The NCBI accession numbers and references of strains used in this figure are listed in Table S3. 


\section{Figure 8}

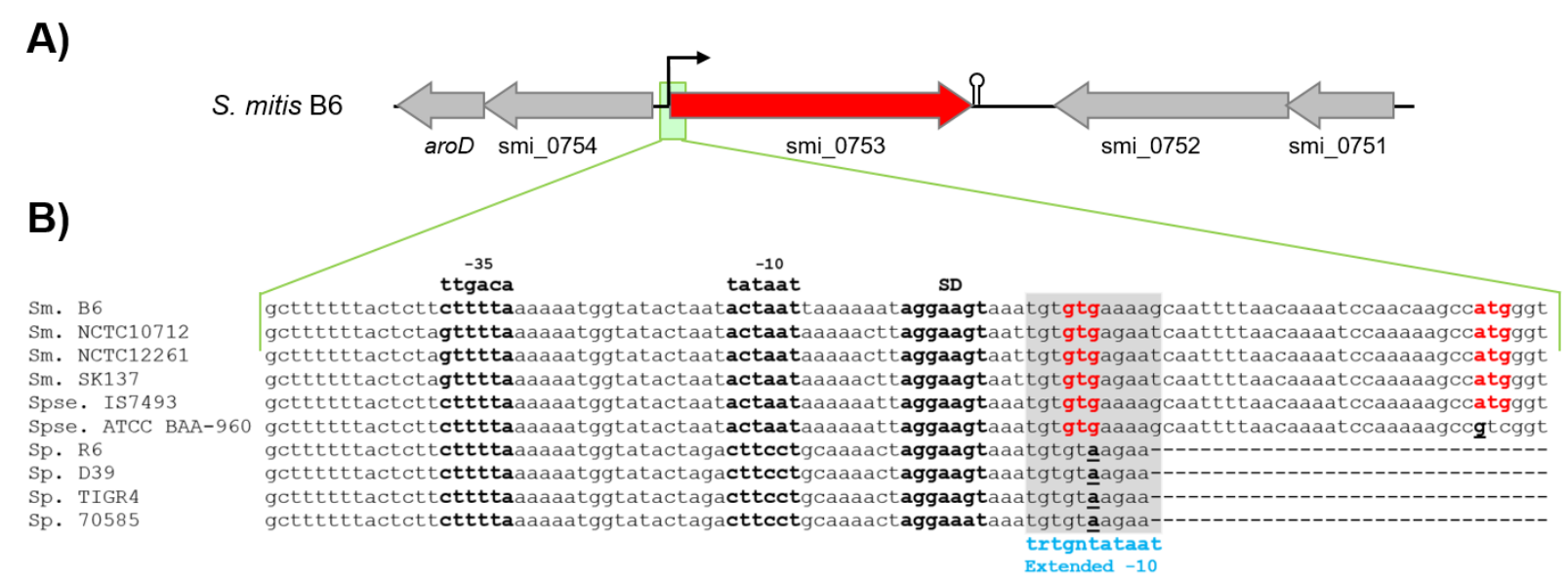

Figure 8. Putative promoter region of the ltaS in S. mitis strain B6. A) Genetic map of the S. mitis B6 genome region with ltaS (smi_0753). The ltaS gene is shown in red, the flanking genes in grey. The small black arrow indicates the putative promoter, the putative transcriptional terminator is shown as stem-loop structure. B) Comparison of the putative promoter region upstream of ltaS (smi_0753) with those of the other S. pneumoniae (Sp.), S. mitis (Sm.) and S. pseudopneumoniae (Spse.) strains. The alignment shows the conserved sequences located upstream of ltaS (smi_0753). The two putative start codons (GTG and ATG) are indicated in red. The putative promoter region (translation start site GTG) is highlighted in bold and the -10 and -35 elements as well as the putative ribosome-binding site (SD) are labelled. The extended -10 region (translation start ATG) is shown in bold within the grey area. The alterations within ATG start codon are underlined and bold. Sequences not present are represented in the alignment by (-). 